\title{
A convenient decontraction procedure of internally contracted state-specific multireference algorithms
}

\author{
Celestino Angelia) \\ Dipartimento di Chimica, Università di Ferrara, Via Borsari 46, I-44100 Ferrara, Italy
}

Carmen J. Calzado

Departamento de Quimica Fisica, Universidad de Sevilla c/ Profesor Garcia Gonzalez, s/n. E-41012 Sevilla, Spain

Renzo Cimiraglia

Dipartimento di Chimica, Università di Ferrara, Via Borsari 46, I-44100 Ferrara, Italy

Jean-Paul Malrieu

IRSAMC, Laboratoire de Physique Quantique, Université Paul Sabatier, 118 route Narbonne, 31062 Toulouse cedex, France

(Received 6 February 2006; accepted 13 April 2006; published online 20 June 2006)

Internally contracted state-specific multireference (MR) algorithms, either perturbative such as CASPT2 or NEVPT2, or nonperturbative such as contracted MR configuration interaction or MR coupled cluster, are computationally efficient but they may suffer from the internal contraction of the wave function in the reference space. The use of a low dimensional multistate model space only offers limited flexibility and is not always practicable. The present paper suggests a convenient state-specific procedure to decontract the reference part of the wave function from a series of state-specific calculations using slightly perturbed zero-order wave functions. The method provides an orthogonal valence bond reading of the ground state and an effective valence Hamiltonian, the excited roots of which are shown to be relevant. The orthogonal valence bond functions can be considered quasidiabatic states and the effective valence Hamiltonian gives therefore the quasidiabatic energies and the electronic coupling among the quasidiabatic states. The efficiency of the method is illustrated in two case problems where the dynamical correlation plays a crucial role, namely, the LiF neutral/ionic avoided crossing and the $\mathrm{F}_{2}$ ground state wave function. (C) 2006 American Institute of Physics. [DOI: 10.1063/1.2202738]

\section{INTRODUCTION}

The development of the theoretical tools for the study of the excited states of molecules remains an active domain of research. The theoretical methods must satisfy a set of criteria: they must be size consistent [ensuring a correct separability of an $(A B)^{*}$ system into $\left.A^{*}+B\right]$, and they must incorporate the leading correlation effects and produce reliable results at a reasonable computational cost. One series of methods [equation-of-motion ${ }^{1}$ (EOM), linear response theory ${ }^{2,3}$ (LRT), coupled cluster with approximations on the fluctuation potential ${ }^{4}$ (CC3)] starts from a correlated description of the ground state (GS) based on a single-reference coupled cluster expansion of the wave function and considers all possible single and double excitations on top of it. The other category of approaches considers that it is possible to build a zero-order description of the excitation process from a limited set of single and double excitations involving a limited number of orbitals. This is close to the traditional and grounded picture of the lowest excited states as obtained from the Hartree-Fock (closed shell) ground state by promoting one (or two) electron(s) from upper lying occupied orbit-

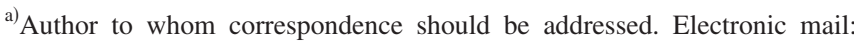
anc@unife.it
}

als to low lying virtual orbitals. Usually it is possible to define an appropriate set of active orbitals and active electrons, defining a complete active space (CAS) to produce a relevant zero-order picture of the excitations. The diagonalization of the corresponding truncated configuration interaction (CI) matrix is expected to produce a well balanced approximate description of the GS $\left(\Psi_{0}\right)$ and of the desired excited $\left(\Psi_{n}\right)$ states. Of course an accurate evaluation of the transition energies (and dipole moments) requires the inclusion of dynamical correlation effects, which are brought by the single and double excitations involving nonactive orbitals acting on the CAS. These hierarchical approaches are called multireference (MR) methods, since they start from a multideterminantal description of the concerned states. Actually these methods also deal with the GS problems for which a single determinantal description is insufficient, as occurs in bond breaking chemical reactions, or magnetic systems involving several open shells.

The post-CAS treatment may be either perturbative [MRPT2 (Refs. 5-19)], variational [MRSDCI (Refs. 20-22)], with size-consistent treatment [MRCEPA (Ref. 23) or, see Ref. 24, MR(SC) (Ref. 2)] or of coupled cluster type [MRCC (Refs. 25-27)]. Again one can subdivide this family of treatments according to their flexibilities and their "contracted character." The aim of the present work is to illustrate 
the possible defects of the internal contraction of MR treatments, as discussed in Sec. II, and to propose a simple procedure, described in Sec. III, which enables one to revise the composition of the desired eigenvector in the model space from a set of state-specific calculations concerning the same state but which slightly differ under small perturbations of elements of the CASCI matrix. The efficiency of the method will be illustrated in two examples where the decontraction is crucial.

\section{POSSIBLE DEFECTS OF THE INTERNAL CONTRACTION OF MULTIREFERENCE TREATMENTS}

Let us call $S_{0}$ the reference subspace and $P_{0}$ the corresponding projector,

$$
P_{0}=\sum_{I \in S_{0}}|I\rangle\langle I|,
$$

where the functions $|I\rangle \in S_{0}$ make up an orthonormal basis set. Usually these vectors are either single determinants or configuration state functions (CSFs). The diagonalization of the CI matrix restricted to the reference space delivers a zero-order wave function of the desired states

$$
\begin{aligned}
& P_{0} \hat{\mathcal{H}} P_{0}\left|\Psi_{m}^{(0)}\right\rangle=E_{m}^{(0)}\left|\Psi_{m}^{(0)}\right\rangle, \\
& \left|\Psi_{m}^{(0)}\right\rangle=\sum_{I \in S_{0}} c_{m, I}^{(0)}|I\rangle .
\end{aligned}
$$

The reference space may be complete or not, it may be obtained from a state-specific or a state-average multiconfigurational self consistent field (MCSCF) calculation or from any set of orbitals. The post-CAS treatment can possibly consider only the interaction of $\Psi_{m}^{(0)}$ with the vectors which do not belong to $S_{0}$. If $S_{0}$ is a CAS of valence character, one frequently says that the diagonalization of $P_{0} \hat{\mathcal{H}} P_{0}$ accounts for the nondynamical correlation, and that the effect of the single and double excitations on top of $\Psi_{m}^{(0)}$ (or on the model space determinants, $|I\rangle)$ will account for the so-called dynamical correlation. One may consider as correction functions (to be referred to as "perturbers" henceforth) either multiconfigurational vectors obtained from $\Psi_{m}^{(0)}$ by the action of the excitation operators $E_{r i}, E_{r i} E_{s j}$ or the (much more numerous) single determinants of the outer space interacting with $\Psi_{m}^{(0)}$ [as done in CIPSI (Refs. 5-7)]. The latter solution is said to be externally decontracted, while the former is termed externally contracted. The NEVPT method, ${ }^{12-14}$ for instance, has introduced various degrees of external contractions. On the other hand, all the methods which do not revise the content of $\Psi_{m}$ in the model space may be called internally contracted. They assume that $\Psi_{m}$ can be written as

$$
\left|\Psi_{m}\right\rangle=\left|\Psi_{m}^{(0)}\right\rangle+\sum_{i \notin S_{0}} c_{m, i}|i\rangle
$$

This may turn out to be rather a crude approximation, since from the second-order of perturbation theory the wave function takes components on all the other eigenstates $\Psi_{n}^{(0)}$ $(n \neq m)$ of the CASCI manifold. It is important at this stage to remark that one may conceive internally decontracted state-specific (SS) formalisms. This requires a state-specific effective Hamiltonian to be built in the model space, which is no longer an effective Hamiltonian in the sense of quasidegenerate perturbation theory $^{28}$ (QDPT) or Bloch's theory, ${ }^{29}$ but an intermediate effective Hamiltonian. ${ }^{30}$ In the field of perturbative approaches the shifted $B_{k}$ technique ${ }^{31}$ is of that type. Some MRCEPA (Ref. 24) or MRCC (Refs. 26 and 27) algorithms are also internally decontracted. An internally decontracted perturbation has been derived ${ }^{15,16}$ from the SSMRCC method of Mahapatra et al. ${ }^{27}$ However, the internal decontraction results in computationally demanding codes and many popular methods take benefit from the simplifications brought by internal contraction.

Among the perturbative methods the CIPSI $\operatorname{code}^{5-7}$ is internally contracted and externally decontracted and so are its numerous variants (see, for instance, Refs. 8 and 17). The popular and efficient CASPT2 method $^{10}$ is both internally and externally contracted, and so is the NEVPT second-order expansion. $^{12-14}$ As mentioned previously, internally contracted MRCI and MRCC codes are numerous and frequently used. The constraint imposed by internal contractions may become severe when the dynamical correlation deeply modifies the content of the wave function in the reference space. Nakano ${ }^{11}$ and Spiegelmann and Malrieu ${ }^{32,33}$ had shown that it may result in spurious double crossings of potential energy curves in the case of weakly avoided crossings (as occurs at long internuclear distances in the GS potential energy curve of LiF). The phenomenon exhibited by CIPSI also appears in the state-specific CASPT2 (Ref. 34) or NEVPT2 calculations. ${ }^{35}$ A solution to that type of problem consists in the construction of an effective Hamiltonian, built on the nearly degenerate states $\Psi_{m}^{(0)}$ and $\Psi_{n}^{(0)}$ which are recombined under the interaction with the outer space. The diagonalization of the effective Hamiltonian based on $\Psi_{m}^{(0)}$ and $\Psi_{n}^{(0)}$, and constructed in the form of the second-order QDPT, produces new vectors and energies which avoid the double curve crossings. This technique leads to the so-called multistate (MS) or quasidegenerate (QD) variants of CIPSI, CASPT2, or NEVPT2. The same improvement may be crucial in some difficult spectroscopic problems, such as the lowest singlet excitation of ethylene, whose Rydberg component is exaggerated at the zero-order CASCI level, and strongly reduced by the $\sigma-\pi$ and dynamical correlation corrections, to the benefit of an essentially valence character. ${ }^{35}$

These MS variants present a rather severe restriction, in that they only proceed to a limited revision of the wave function given that the dimension of the reference space is usually larger than the dimension of the model space. Moreover the technique may be impracticable in many problems.

- Consider, for instance, the calculation of the potential energy surface (PES) of a GS $\Psi_{0}$ which presents a weakly avoided crossing with $\Psi_{1}$ for some nuclear coordinates. One can then consider a two-state treatment concerning both $\Psi_{0}$ and $\Psi_{1}$, but if (as usually occurs) $\Psi_{1}$ presents a weakly avoided crossing with $\Psi_{2}$ for other domains of the nuclear coordinates, the two-state treatment will be affected in this region. Including $\Psi_{2}$ in the model space can even worsen the situation since the number of avoided crossing usually increases when 
one goes to higher and higher excited states. Using different model spaces in different domains of the nuclear coordinates results in discontinuities of the PES. Hence a state-specific technique which would be able to follow a given state through weakly avoided crossings would be a valuable tool.

- The change of the wave function in the reference space from its zero-order approximation to its exact content might not necessarily be seen as a rotation between two spectroscopic states, $\Psi_{m}$ and $\Psi_{n}$. A very simple and well documented problem of this type concerns the magnetic binuclear $\mathrm{Cu}\left(d^{9}\right)$ complexes in the two lowest states $^{36}$ (singlet and triplet). Each metallic center, $A$ (and $B$ ), bears an unpaired electron in a magnetic singly occupied orbital, $a$ (and $b$ ). The minimal CAS involves two electrons in two orbitals and is composed, in a valence bond language, of the neutral valence bond (VB) determinants $\mid$ core $a \bar{b} \mid$ and $\mid$ core $b \bar{a} \mid$, and the ionic VB determinants $\mid$ core $a \bar{a} \mid$ and $\mid$ core $b \bar{b} \mid$. These determinants can be combined obtaining a neutral singlet $\quad \Psi_{S}^{N}=(\mid$ core $a \bar{b}|+|$ core $b \bar{a} \mid) / \sqrt{2}, \quad$ a neutral triplet $\Psi_{T}^{N}=(\mid$ core $a \bar{b}|-|$ core $b \bar{a} \mid) / \sqrt{2}$, and two ionic singlets $\quad \Psi_{S}^{I, 1}=(\mid$ core $a \bar{a}|+|$ core $b \bar{b} \mid) / \sqrt{2}$ and $\Psi_{S}^{I, 2}$ $=(\mid$ core $a \bar{a}|-|$ core $b \bar{b} \mid) / \sqrt{2}$. The interaction between the neutral and ionic singlets is responsible for the generally observed antiferromagnetic preference, i.e., the singlet character of the ground state. However, a precise evaluation of the energy difference between the low lying singlet and triplet states requires an accurate consideration of the dynamical correlation effects. The dynamical polarization of the ionic VB structures $A^{-} B^{+}$ and $A^{+} B^{-}$stabilizes their effective energies, and increases their weight in the wave function and their impact on the singlet state energy. Uncontracted CI calculations show that the ratio of the coefficients of the ionic VB determinants over the coefficients of the neutral ones is multiplied by a factor 4 when going from the CASCI to extensive CI. A contracted treatment, starting from functions $\Psi_{0}^{S}$, which dramatically underestimate the ionic VB component of the singlet wave function, should result (except for fortuitous cancellations of errors) in an underestimation of the singlet state energy stabilization. This precise problem will be studied in detail in a further work, ${ }^{37}$ but one should notice here that it is impracticable to apply the MS variant of perturbative techniques to these problems. Actually the ionic VB component of the reference space does not generate an accessible and well defined spectroscopic state as it is embedded in a multitude of ligand/metal charge transfer states. Any perturbative approach of the corresponding eigenenergy is liable to diverge.

- To a lower extent the same problem is present in some covalent bonded systems such as $F_{2}$, where the two electrons of the $\sigma$ bond are surrounded by six polarizable electron pairs. The dynamic response of these pairs to the fluctuation of the electric field induced by the movement of the two electrons of the $\sigma$ bond is crucial in obtaining a reasonable dissociation energy of that simple bond. ${ }^{38-40}$

\section{A SIMPLE STATE-SPECIFIC DECONTRACTION PROCEDURE}

Let us expand the zero-order MR function $\Psi_{m}^{(0)}$ on a set of functions which define a basis of the reference $N$-dimensional $S_{0}$ space,

$$
\left|\Psi_{m}^{(0)}\right\rangle=\sum_{I \in S_{0}} c_{m, I}^{(0)}|I\rangle
$$

The functions $\{|I\rangle\}$ can be determinants, CSFs, or general multireference functions. The second-order energy correction from the contracted wave function is

$$
E_{m}^{(2)}=\sum_{I, J}^{N} \sum_{\alpha} c_{m, I}^{(0)} \frac{\langle I|\hat{\mathcal{H}}| \alpha\rangle\langle\alpha|\hat{\mathcal{H}}| J\rangle}{E_{m}^{(0)}-E_{\alpha}^{(0)}} c_{m, J}^{(0)}
$$

where $\alpha$ runs on the outer space (which is made of single determinants in CIPSI, linear combinations of determinants in externally contracted methods). The expression defines a dressing operator $\hat{\Delta}^{m}$,

$$
\hat{\Delta}^{m}=\sum_{\alpha} \frac{\hat{\mathcal{H}}|\alpha\rangle\langle\alpha| \hat{\mathcal{H}}}{E_{m}^{(0)}-E_{\alpha}^{(0)}},
$$

whose matrix elements in the $\{|I\rangle\}$ basis are

$$
\left\langle I\left|\hat{\Delta}^{m}\right| J\right\rangle=\sum_{\alpha} \frac{\langle I|\hat{\mathcal{H}}| \alpha\rangle\langle\alpha|\hat{\mathcal{H}}| J\rangle}{E_{m}^{(0)}-E_{\alpha}^{(0)}} .
$$

Then

$$
E_{m}^{(2)}=\left\langle\Psi_{m}^{(0)}\left|\hat{\Delta}^{m}\right| \Psi_{m}^{(0)}\right\rangle,
$$

with the $m$ dependence being due to the energy denominators. The dressing operator, introduced in the $B_{k}$ technique, ${ }^{31}$ is state specific and then it would be significantly different for another state $\Psi_{n}^{(0)}$.

The decontracted energy and wave function will be given by the diagonalization of the operator $P_{0}\left(\hat{\mathcal{H}}+\hat{\Delta}^{m}\right) P_{0}$ in the $\{|I\rangle\}$ basis,

$$
P_{0}\left(\hat{\mathcal{H}}+\hat{\Delta}^{m}\right) P_{0}\left|\widetilde{\Psi}_{m}^{(0)}\right\rangle=\widetilde{E}_{m}^{(0)}\left|\widetilde{\Psi}_{m}^{(0)}\right\rangle .
$$

The direct calculation of the matrix elements of the operator defined in Eq. (7) can be straightforward, as, for instance, in the diagrammatic implementation of CIPSI (Refs. 6 and 41) (using as basis $\{|I\rangle\}$ the reference determinants), or may present substantial difficulties, as happens for NEVPT2 (again when the $|I\rangle$ 's are the reference determinants). In this approach one can apply suitable operators to the zero-order wave function as a whole, strongly reducing the computational effort ${ }^{13,14}$ for the calculation of the second-order correction to the energy. However, such an advantage disappears if in the perturbation formula the zero-order wave function is replaced by the determinants $\{|I\rangle\}$ [Eq. (8)] and this makes almost impracticable the direct use of this formula with determinants (or CSFs) as basis. The use of the eigenstates of 
$\hat{\mathcal{H}}$ as the $\{|I\rangle\}$ basis in the reference space solves these difficulties. However, the adoption of this strategy would imply exploiting the full dimensionality of the reference space leading, in most cases, to a very expensive calculation. Such an effort is expected to be excessive when large reference spaces are used, given that the internal revision of the wave function usually depends on the rotation of $\Psi_{m}^{(0)}$ with few wave functions of the reference space. When the direct use of Eq. (8) is difficult or too expensive, one can resort to a numerical approach, which in some aspects resembles the finite-field strategy for the calculations of molecular properties (for instance, the electric dipole). This approach is presented in the following.

Suppose that one perturbs the system and computes the eigenenergy of the state under study from a slightly different vector $\left|\Psi_{m, k}^{(0)}\right\rangle=\sum_{I}^{N} c_{m, I}^{(0), k}|I\rangle$. This would produce a slightly different value of $E_{m}^{(2)}$, namely, $E_{m}^{(2), k}$,

$$
E_{m}^{(2), k}=\sum_{I, J}^{N} c_{m, I}^{(0), k}\left\langle I\left|\hat{\Delta}_{k}^{m}\right| J\right\rangle c_{m, J}^{(0), k} .
$$

If one maintains the same value for the energy denominators, $E_{m}^{(0)}-E_{\alpha}^{(0)}=E_{m}^{(0), k}-E_{\alpha}^{(0), k}$, and supposes that the perturbers $|\alpha\rangle$ do not depend on $\Psi_{m}^{(0)}$, then $\hat{\Delta}_{k}^{m}=\hat{\Delta}^{m}$. In these conditions, if the reference space involves $N$ determinants and $N^{2}$ matrix elements, $N^{2}$ state-specific calculations from slightly distinct vectors $\Psi_{m, k}^{(0)}$ will produce sufficient information to solve the linear equations (11) for the $N^{2}$ matrix elements of the dressing operator $\hat{\Delta}^{m}$ from the $N^{2}$ values $E_{m}^{(2), k}$. Since from a statespecific procedure one expects the dressing matrix to be symmetrical, $N(N+1) / 2$ calculations are sufficient. One may also assume that a diagonal form of the dressing matrix is sufficient and only perform $N$ state-specific calculations to determine the quantities $\left\langle I\left|\hat{\Delta}^{m}\right| I\right\rangle$.

In all cases the decontracted energy will be given by the diagonalization of the matrix $P_{0}\left(\hat{\mathcal{H}}+\hat{\Delta}^{m}\right) P_{0}$ according to Eq. (10).

A control of the relevance of the selected procedure (diagonal, Hermitian) will be given by testing the stability of the matrix elements of $\hat{\Delta}^{m}$ and of the eigenenergy $\widetilde{E}_{m}^{(0)}$ under additional changes of $\Psi_{m, k}^{(0)}$.

So far the procedure is general and it can be applied as well to contracted MRCI or MRCC procedures which in- clude higher order perturbative corrections. In practice many codes require, in order to have computational benefits, that $\Psi_{m}^{(0)}$ is an eigenvector of the CASCI matrix. In that case one may introduce slight modification of the elements of the CASCI matrix which should not modify the numerators in Eq. (6) and should lead to only minor changes in the energy denominators. The procedure is then approximate and its stability with respect to these changes of the matrix elements between active orbitals has to be checked carefully.

One may, for instance, introduce a small energy change of the monoelectronic energy of an active orbital. This defines a perturbation operator $\mathcal{V}$. If $\Psi_{m}^{(0)}$ was the eigenvector of the CASCI matrix before perturbation, the perturbation $\mathcal{V}$ introduces a small modification of the eigenvector,

$$
\Psi_{m}^{(0)}(\mathcal{V})=\lambda \Psi_{m}^{(0)}+\mu \Psi_{m}^{\perp}(\mathcal{V}),
$$

where $\left\langle\Psi_{m}^{\perp}(\mathcal{V}) \mid \Psi_{m}^{(0)}\right\rangle=0$. In the limit of vanishing perturbation $\Psi_{m}^{\perp}(\mathcal{V})$ does not depend on the amplitude of $\mathcal{V}$ : for a finite perturbation this becomes an approximation and one can suppose that it is better grounded if only two states/ $\mathrm{CSF}$ /VB forms are supposed to play a role in the decontraction procedure. Changing the magnitude of the perturbation $(\mathcal{V} \rightarrow k \mathcal{V})$ and supposing the invariance of $\Psi_{m}^{\perp}$, one has another expression for $\Psi_{m}^{(0)}$,

$$
\Psi_{m}^{(0)}(k \mathcal{V})=\lambda_{k} \Psi_{m}^{(0)}+\mu_{k} \Psi_{m}^{\perp} .
$$

The hypothesis of the invariance of $\Psi_{m}^{\perp}$ with the magnitude of the perturbation can be easily verified computing the overlap $\left\langle\Psi_{m}^{\perp}(\mathcal{V}) \mid \Psi_{m}^{\perp}(k \mathcal{V})\right\rangle$ and verifying whether it is close to 1 . The calculation of $E_{m}^{(2)}, E_{m}^{(2)}(\mathcal{V})$ and $E_{m}^{(2)}(k \mathcal{V})$ will allow us to define an effective Hamiltonian in terms of $\Psi_{m}^{(0)}$ and $\Psi_{m}^{\perp}$, with the equations,

$$
\begin{aligned}
& E_{m}^{(2)}=\left\langle\Psi_{m}^{(0)}\left|\hat{\Delta}^{m}\right| \Psi_{m}^{(0)}\right\rangle, \\
& E_{m}^{(2)}(\mathcal{V})= \lambda^{2}\left\langle\Psi_{m}^{(0)}\left|\hat{\Delta}^{m}\right| \Psi_{m}^{(0)}\right\rangle+2 \lambda \mu\left\langle\Psi_{m}^{(0)}\left|\hat{\Delta}^{m}\right| \Psi_{m}^{\perp}\right\rangle \\
&+\mu^{2}\left\langle\Psi_{m}^{\perp}\left|\hat{\Delta}^{m}\right| \Psi_{m}^{\perp}\right\rangle, \\
& E_{m}^{(2)}(k \mathcal{V})= \lambda_{k}^{2}\left\langle\Psi_{m}^{(0)}\left|\hat{\Delta}^{m}\right| \Psi_{m}^{(0)}\right\rangle+2 \lambda_{k} \mu_{k}\left\langle\Psi_{m}^{(0)}\left|\hat{\Delta}^{m}\right| \Psi_{m}^{\perp}\right\rangle \\
&+\mu_{k}^{2}\left\langle\Psi_{m}^{\perp}\left|\hat{\Delta}^{m}\right| \Psi_{m}^{\perp}\right\rangle .
\end{aligned}
$$

Hence

$$
\begin{aligned}
& \left\langle\Psi_{m}^{(0)}\left|\hat{\Delta}^{m}\right| \Psi_{m}^{(0)}\right\rangle=E_{m}^{(2)}, \\
& \left\langle\Psi_{m}^{(0)}\left|\hat{\Delta}^{m}\right| \Psi_{m}^{\perp}\right\rangle=\left.\frac{\mu_{k}^{2} E_{m}^{(2)}(\mathcal{V})-\mu^{2} E_{m}^{(2)}(k \mathcal{V})+\left(\mu^{2} \lambda_{k}^{2}-\lambda^{2} \mu_{k}^{2}\right) E_{m}^{(2)}}{2 \mu \mu_{k}\left(\lambda \mu_{k}-\mu \lambda_{k}\right)}\right|_{\lim _{\mathcal{V} \rightarrow 0}}, \\
& \left\langle\Psi_{m}^{\perp}\left|\hat{\Delta}^{m}\right| \Psi_{m}^{\perp}\right\rangle=\left.\frac{\lambda_{k} \mu_{k} E_{m}^{(2)}(\mathcal{V})-\lambda \mu E_{m}^{(2)}(k \mathcal{V})+\lambda \lambda_{k}\left(\mu \lambda_{k}-\lambda \mu_{k}\right) E_{m}^{(2)}}{\mu \mu_{k}\left(\mu \lambda_{k}-\lambda \mu_{k}\right)}\right|_{\lim _{\mathcal{V} \rightarrow 0}} .
\end{aligned}
$$


The limit $\mathcal{V} \rightarrow 0$ in Eqs. (18) and (19) has been introduced in order to stress that these equations are exact in the limit of small perturbations. On the other hand, in order to avoid numerical instability problems in these equations, $\mathcal{V}$ must be sufficiently large to assure a nonvanishing value for $\mu$ and $\mu_{k}$.

As a further approximation, one can suppose that for a small perturbation $\mu_{k}=k \mu$, and therefore $\lambda_{k}=\sqrt{1-k^{2}+k^{2} \lambda^{2}}$ for the normalization constraint. With this approximation, Eqs. (18) and (19) become particularly compact if $k=-1$. In this case $\mu_{k}=-\mu$ and $\lambda_{k}=\lambda$ so that

$$
\begin{aligned}
& \left\langle\Psi_{m}^{(0)}\left|\hat{\Delta}^{m}\right| \Psi_{m}^{\perp}\right\rangle=\left.\frac{E_{m}^{(2)}(\mathcal{V})-E_{m}^{(2)}(-\mathcal{V})}{4 \lambda \mu}\right|_{\lim _{\mathcal{V} \rightarrow 0},}, \\
& \left\langle\Psi_{m}^{\perp}\left|\hat{\Delta}^{m}\right| \Psi_{m}^{\perp}\right\rangle=\left.\frac{E_{m}^{(2)}(\mathcal{V})+E_{m}^{(2)}(-\mathcal{V})-2 \lambda^{2} E_{m}^{(2)}}{2 \mu^{2}}\right|_{\lim _{\mathcal{V} \rightarrow 0} .} .
\end{aligned}
$$

As seen from Eqs. (12), (20), and (21) the procedure may face numerical instability problems when the amplitude of the perturbation tends to zero, since the denominators (and the numerators) tend to zero in the last two equations. One must therefore check the behavior of the effective Hamiltonian matrix, in order to avoid entering in the instability regime. This instability may occur for different values of the perturbation for different approaches. In the next section we report an example where the strongly contracted and the partially contracted versions of the NEVPT treatment show a different behavior with respect to this aspect. Giving a few values to the perturbation amplitude and checking the stability of the dressing operator provides consistent results. Notice that a similar procedure is used regarding the amplitudes of the level shifts introduced in CASPT2 calculations to avoid the intruder state artifacts. One has to explore the domain of level shifts giving a consistent linear dependence of the energies with respect to the amplitude of the level shift and eventually extrapolate to zero from this domain.

In this approach the internal decontraction is originated by the mixing of $\Psi_{m}^{(0)}$ with only one vector belonging to the reference space, thus strongly reducing the number of elements of the dressing matrix which have to be computed. Of course this may be not enough if more than two states/ $\mathrm{CSF}$ /VB structures are supposed to play a role in the revision of $\Psi_{m}^{(0)}$. In this case one may conceive several perturbation operators $\mathcal{V}$. Another perturbation $\mathcal{V}^{\prime}$ may introduce an additional vector $\Psi_{m}^{* \prime}$, which can be orthogonalized to $\Psi_{m}^{(0)}$ and $\Psi_{m}^{\perp}$, in order to build an effective Hamiltonian of dimension three.

If the procedure is externally contracted, the perturbing vectors $|\alpha\rangle$ depend on $\Psi_{m}^{(0)}$ and the energy numerators in Eq. (7) are not invariant with the changes of $\Psi_{m}^{(0)}$. The proposed procedure becomes somewhat more approximate, but again performing more calculations than strictly required enables one to check the numerical stability of the results.

The procedure described in this section will be referred to with the acronym FDD-MR, from finite difference-based decontraction of contracted multireference methods.

\section{NUMERICAL TEST STUDIES}

\section{A. The LiF molecule}

The avoided crossing of the neutral and ionic states of the LiF molecule is a case where internally contracted multireference methods are known to fail. The origin of this difficulty can be understood noting that, for nuclear geometries near the avoided crossing, the relative weights of the ionic $\left(\mathrm{Li}^{+} \mathrm{F}^{-}\right)$and neutral $(\mathrm{Li} \cdot \cdot \mathrm{F})$ forms in the first two electronic singlet wave functions strongly depend upon the level at which the electronic correlation is taken into account. This is due to the fact that the correlation energy is larger for the ionic form than for the neutral one, therefore an unbalanced description of the electronic correlation modifies the relative energy of a form with respect to the other and consequently changes their coefficients in the wave functions. The problem is particularly evident at the nuclear geometries where the two forms are quasidegenerate.

The difficulty to describe this molecule appears, for instance, in the valence CASSCF (two electrons in two orbitals) method, where the lack of the dynamical electronic correlation makes the ionic form too high in energy with respect to the neutral form (note, however, that at short internuclear distances the ionic form is lower than the neutral one). As a consequence, the weight of the ionic form is too small in the GS and too large in the excited state, the crossing is found at too short an internuclear distance and with too large an energy separation between the two states (see, for instance, Ref. 35). If the CASSCF wave functions are used as starting point in internally contracted state-specific multireference algorithms (either variational or perturbational), this bias cannot be corrected. In the case of a second-order perturbation approach a double crossing is found instead of the avoided crossing. ${ }^{11,35,42}$

The avoided crossing is also found in other alkali halides, ${ }^{43}$ but the LiF molecule is particularly interesting given its small size and that full configuration interaction (FCI) studies have been published. ${ }^{44,45}$ For these reasons this system has often been used to test new methods developed with the aim to face, in the case of quasidegenerate states, the contraction/decontraction problem. ${ }^{11,35,42,44-47}$

In the present study, the $\operatorname{Li}(9 s 5 p) /[4 s 2 p]$ and $\mathrm{F}(9 s 6 p 1 d) /[4 s 3 p 1 d]$ basis sets, employed by Bauschlicher and Langhoff ${ }^{44}$ and by Legeza et al. ${ }^{45}$ for FCI studies on the first two ${ }^{1} \Sigma^{+}$states, have been used. The zero-order wave functions have been obtained by a state-averaged CASSCF procedure, involving the GS and the first ${ }^{1} \Sigma^{+}$excited state, using the MOLCAS package. ${ }^{48}$ Two active spaces have been used: the first contains the $\mathrm{Li} 2 s$ and $\mathrm{F} 2 p_{z}$ valence orbitals and electrons (CASSCF 2/2), while the second space is built from the previous one adding the $\mathrm{F} 2 p_{x}$ and $2 p_{y}$ orbitals and electrons plus two $\mathrm{F} 2 p_{x}^{\prime}$ and $2 p_{y}^{\prime}$ correlating orbitals (CASSCF 6/6). Figure 1 shows the FCI, CASSCF $2 / 2$, and CASSCF 6/6 energies of the two noncrossing states: as already discussed, one notes that at the CASSCF level the crossing is at too short an internuclear distance and with too large an energy splitting with respect to FCI, with CASSCF 6/6 being slightly closer to FCI than CASSCF $2 / 2$.

If the CASSCF zero-order description is perturbatively 


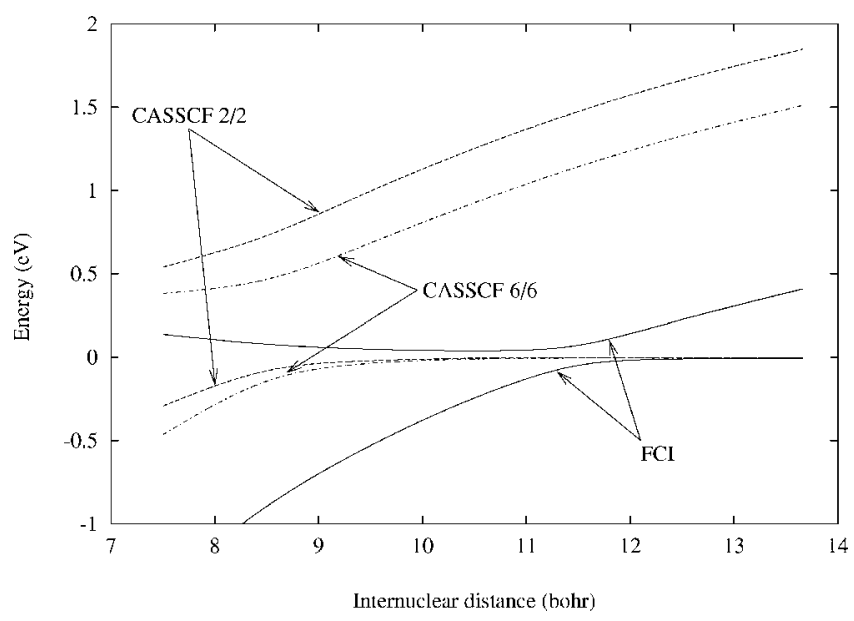

FIG. 1. Potential energy curves (eV) computed at the CASSCF $2 / 2$, CASSCF 6/6, and FCI level for the first two ${ }^{1} \Sigma^{+}$states of LiF in the avoided crossing region.

corrected at second-order, the curves show a double crossing instead of an avoided crossing, ${ }^{11,35,42}$ as shown in Fig. 2, for the case of the state-specific strongly contracted (SC) and partially contracted (PC) variants of NEVPT2 applied to the CASSCF $2 / 2$ wave functions (a similar behavior is found with CASSCF 6/6). In these calculations and in the following ones, the $1 \sigma, 2 \sigma$, and $3 \sigma$ orbitals are kept frozen (at the ground state CASSCF 2/2 level) in the correlation treatment in order to compare with the FCI results.

In order to apply the FDD-MR decontraction procedure described in Sec. III let us note, first of all, that the CASCI $2 / 2$ space is spanned by three CSFs, one neutral $(\mathrm{Li} \cdot+\cdot \mathrm{F})$ and the other two ionic $\left(\mathrm{Li}^{+}+\mathrm{F}^{-}\right.$and $\left.\mathrm{Li}^{-}+\mathrm{F}^{+}\right)$. Among these CSFs the last one has a vanishing weight in the two states under study. Indeed, in the range of internuclear distances here considered (from 6.5 to 13.70 bohrs), the square of its coefficient is always lower than $10^{-6}$. For this small active space one can therefore focus the attention on the two relevant CSFs, hereafter called $|I\rangle$ (ionic) and $|N\rangle$ (neutral).

The three elements of the dressing matrix [Eq. (7)] in the basis $\{|I\rangle,|N\rangle\}$ are obtained by inverting the expression re-

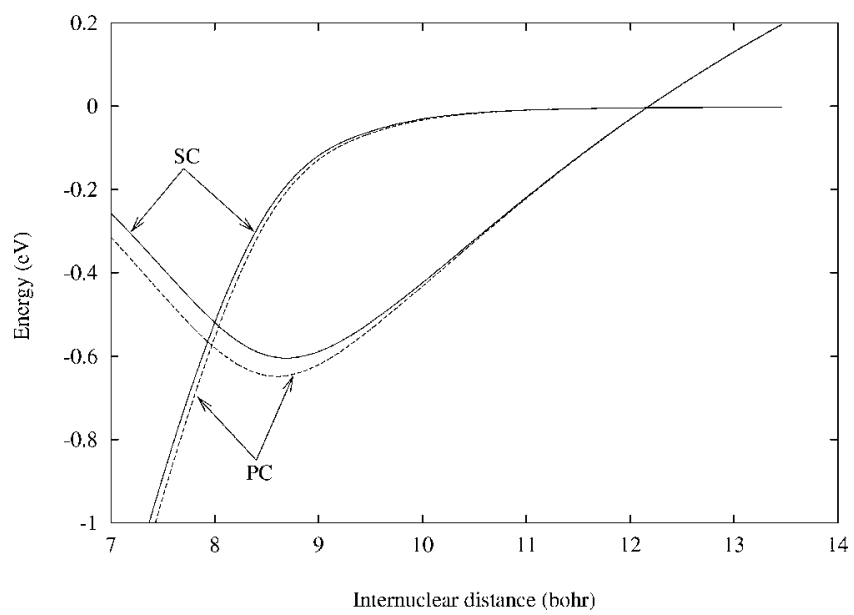

FIG. 2. Potential energy curves (eV) computed at the CASSCF $2 / 2+$ SC-NEVPT2, and CASSCF $2 / 2+$ PC-NEVPT2 level for the first two ${ }^{1} \Sigma^{+}$states of $\mathrm{LiF}$ in the avoided crossing region.

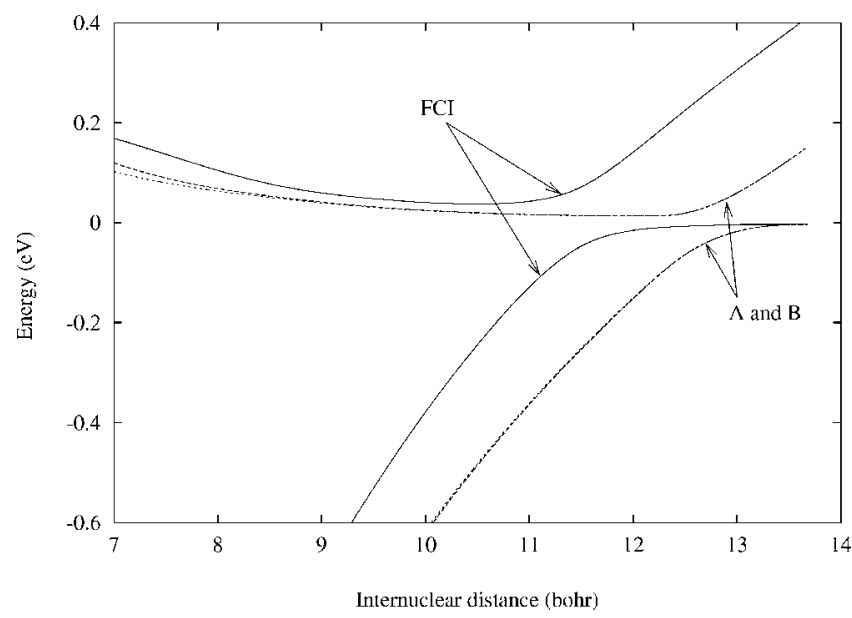

FIG. 3. Adiabatic potential energy curves (eV) for the first two ${ }^{1} \Sigma^{+}$states of $\mathrm{LiF}$ computed with the FDD-MR procedure at the CASSCF 2/2+SC-NEVPT2 level. A and B indicate a decontraction based on different choices of $\gamma$ (see text). The FCI curves are also reported for comparison.

ported in Eq. (11) evaluated for three different zero-order wave functions $\Psi_{m, k}^{(0)}$ of the ground state. These wave functions are obtained using the state-averaged CASSCF $2 / 2$ orbitals in a CASCI calculation of the GS where the oneelectron energy of the Li $2 s$ orbital is shifted by an amount $\gamma$. In order to test the stability of the decontraction algorithm with respect to the computational parameters, the dressing matrix has been obtained with two approaches (indicated hereafter with the letters $\mathrm{A}$ and $\mathrm{B}$ ):

(a) $\gamma=0.0, \pm 0.10 \rightarrow$ "A;"

(b) $\gamma=0.0, \pm 0.20 \rightarrow$ "B."

The results of such an approach are reported, for both choices of $\gamma$ values, in Figs. 3 and 4 for the SC and PCNEVPT2 calculations, respectively. All the energies are reported in $\mathrm{eV}$ using as zero the energy of the GS at 13.70 bohrs. One promptly notes that the unphysical behavior of the uncontracted NEVPT2 curves has disappeared and that a correct description of the avoided crossing is restored. Indeed, even if the avoided crossing appears at a slightly too

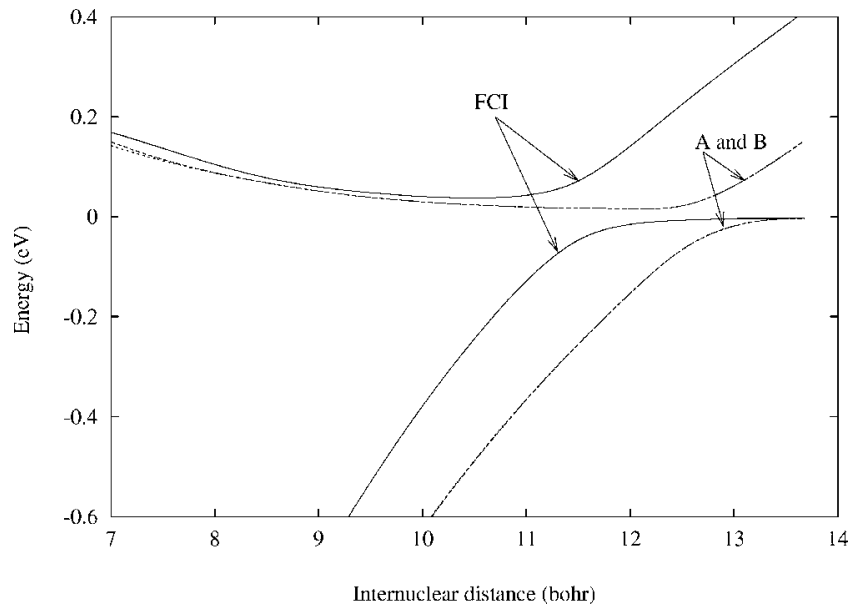

FIG. 4. Adiabatic potential energy curves (eV) for the first two ${ }^{1} \Sigma^{+}$states of $\mathrm{LiF}$ computed with the FDD-MR procedure at the CASSCF $2 / 2+$ PC-NEVPT2 level. A and B indicate a decontraction based on different choices of $\gamma$ (see text). The FCI curves are also reported for comparison. 
TABLE I. Internuclear geometry (bohr) at which the neutral and ionic structures of the LiF molecule are degenerate at the CASSCF 2/2, NEVPT2, FDD-MR/NEVPT2, and FCI level. For the NEVPT2 approach two crossing points are found (see text).

\begin{tabular}{lc}
\hline \hline Method & Crossing distance \\
\hline CASSCF & 7.25 \\
FCI & $11.42^{\mathrm{a}}$
\end{tabular}

\begin{tabular}{lcc} 
& SC-NEVPT2 & PC-NEVPT2 \\
\hline NEVPT2 & $8.00,12.20$ & $7.97,12.20$ \\
FDD-MR/NEVPT2 (A) & 12.80 & 12.80 \\
FDD-MR/NEVPT2 (B) & 12.80 & 12.80 \\
\hline \hline
\end{tabular}

${ }^{\mathrm{a}}$ Distance at which the two states have the same electric dipole, Ref. 35.

long internuclear distance and with a slightly too small energy separation, the improvement with respect to the CASSCF and uncontracted NEVPT2 description is evident. For the sake of clarity, the internuclear distance at which the neutral and ionic structures are degenerate are reported in Table I at the CASSCF, NEVPT2, and FDD-MR/NEVPT2 level. For both variants of NEVPT2 the A and B curves are almost superimposed on each other: tests have been performed with larger and smaller values for $\gamma$, always obtaining the same behavior. In this case the stability of the results with respect to the values of $\gamma$ used in the FDD-MR approach is related to the small dimensionality of the CAS space which imposes that the GS wave function has essentially only two components for all values of $\gamma$. Moreover one notes that for both choices of $\gamma$ values SC-NEVPT2 and PC-NEVPT2 give very similar results.

In Table II the elements of the Hamiltonian operator (at the CASSCF $2 / 2$ level) and of the dressing operator (at the SC-NEVPT2 and PC-NEVPT2 level with the A choice of $\gamma$ values) on the $|N\rangle$ and $|I\rangle$ basis are reported. From this table some considerations can be drawn: (a) the diagonal elements of the dressing matrix are almost constant in the range of internuclear coordinates here considered; (b) the off-diagonal element of the dressing matrix shows a sizable variation, but it is small in the crossing region; and (c) the lowering of the ionic form effected by the perturbation approach is much larger than the one of the neutral form.

The results reported in Table II allow one to obtain, apart from the adiabatic curves reported in Figs. 3 and 4 the ionic and neutral diabatic potential energy curves (adding the appropriate diagonal elements of $\hat{\mathcal{H}}$ to those of $\hat{\Delta}$ ) and the electronic coupling among the diabatic states (adding the two off-diagonal elements of $\hat{\mathcal{H}}$ and $\hat{\Delta}$ ). From these data one can easily compute the nonadiabatic coupling (retaining only the "coefficient mixing" contribution, see Refs. 49 and 50). All such information is of great relevance, for instance, in dynamical studies. It is relevant to note that they are obtained within a state-specific procedure: this makes a difference with respect to other "diabatization methods" which need the simultaneous description of more than one state. In order to better emphasize the large effect of the perturbation on the diabatic states, the CASSCF $2 / 2$ and FDD-MR/SC-NEVPT2 diabatic energy curves are reported in Fig. 5 (a similar behavior is found for FDD-MR/PC-NEVPT2).

The disagreement of the FDD-MR/SC-NEVPT2 and FDD-MR/PC-NEVPT2 results with the FCI ones is due to an overshooting of the perturbation approach in estimating the dynamical correlation energy of the ionic form thus leading to a crossing between the two diabatic curves at too long an internuclear distance. This behavior originates from the low quality of the zero-order wave function, and cannot be attributed to the choice of $\gamma$ values here proposed.

In order to improve the zero-order description of the wave functions, CASSCF $6 / 6$ calculations have been performed. This active space (or a larger one) has been used in the previously published works ${ }^{34,35,47,51,52}$ and contains 55 CSFs (112 determinants). The use of all these functions as basis for the decontraction procedure is certainly excessive. Therefore Eqs. (17)-(19) have been used for the construction of a $2 \times 2$ dressing matrix on the basis of the zero-order

TABLE II. Matrix elements of the Hamiltonian operator (at the CASSCF level) and of the dressing operator (at the SC-NEVPT2 and PC-NEVPT2 level) on the ionic and neutral VB structures for the LiF molecule as a function of the internuclear distance. The matrix elements of the dressing operator are obtained by shifting the one-electron energy of the Li $2 s$ orbital by $0,0.1$, and -0.1 hartree (see text). Energies in hartree, and distance in bohr.

\begin{tabular}{|c|c|c|c|c|c|c|c|c|c|}
\hline \multirow[b]{2}{*}{$R$} & \multicolumn{3}{|c|}{ CASSCF } & \multicolumn{3}{|c|}{ SC-NEVPT2 } & \multicolumn{3}{|c|}{ PC-NEVPT2 } \\
\hline & $\langle N|\hat{\mathcal{H}}| N\rangle$ & $\langle I|\hat{\mathcal{H}}| I\rangle$ & $\langle N|\hat{\mathcal{H}}| I\rangle$ & $\langle N|\hat{\Delta}| N\rangle$ & $\langle I|\hat{\Delta}| I\rangle$ & $\langle N|\hat{\Delta}| I\rangle$ & $\langle N|\hat{\Delta}| N\rangle$ & $\langle I|\hat{\Delta}| I\rangle$ & $\langle N|\hat{\Delta}| I\rangle$ \\
\hline 4.50 & -106.772474 & -106.874937 & 0.026578 & -0.134039 & -0.190990 & -0.008437 & -0.133025 & -0.191532 & -0.006586 \\
\hline 5.50 & -106.779821 & -106.833029 & 0.026256 & -0.133904 & -0.192565 & -0.008955 & -0.132363 & -0.193102 & -0.006381 \\
\hline 6.50 & -106.784985 & -106.803485 & 0.021278 & -0.131827 & -0.192613 & -0.007268 & -0.130875 & -0.192998 & -0.004820 \\
\hline 7.50 & -106.787623 & -106.782522 & 0.015090 & -0.130469 & -0.192460 & -0.004923 & -0.129997 & -0.192718 & -0.002935 \\
\hline 8.50 & -106.788774 & -106.766674 & 0.009954 & -0.129864 & -0.192348 & -0.002773 & -0.129691 & -0.192566 & -0.001504 \\
\hline 9.50 & -106.789250 & -106.754132 & 0.006306 & -0.129684 & -0.192400 & -0.001534 & -0.129633 & -0.192569 & -0.000856 \\
\hline 10.00 & -106.789369 & -106.748788 & 0.004969 & -0.129646 & -0.192452 & -0.001156 & -0.129618 & -0.192588 & -0.000666 \\
\hline 10.50 & -106.789447 & -106.743947 & 0.003894 & -0.129619 & -0.192501 & -0.000877 & -0.129604 & -0.192607 & -0.000520 \\
\hline 11.00 & -106.789496 & -106.739548 & 0.003037 & -0.129601 & -0.192537 & -0.000666 & -0.129592 & -0.192616 & -0.000405 \\
\hline 11.25 & -106.789514 & -106.737498 & 0.002680 & -0.129594 & -0.192548 & -0.000581 & -0.129588 & -0.192615 & -0.000358 \\
\hline 11.50 & -106.789530 & -106.735537 & 0.002360 & -0.129588 & -0.192554 & -0.000507 & -0.129584 & -0.192611 & -0.000316 \\
\hline 12.00 & -106.789552 & -106.731867 & 0.001827 & -0.129582 & -0.192549 & -0.000385 & -0.129580 & -0.192589 & -0.000243 \\
\hline 12.50 & -106.789568 & -106.728494 & 0.001410 & -0.129581 & -0.192526 & -0.000292 & -0.129580 & -0.192552 & -0.000187 \\
\hline 13.70 & -106.789588 & -106.721426 & 0.000745 & -0.129591 & -0.192426 & -0.000149 & -0.129591 & -0.192436 & -0.000096 \\
\hline
\end{tabular}




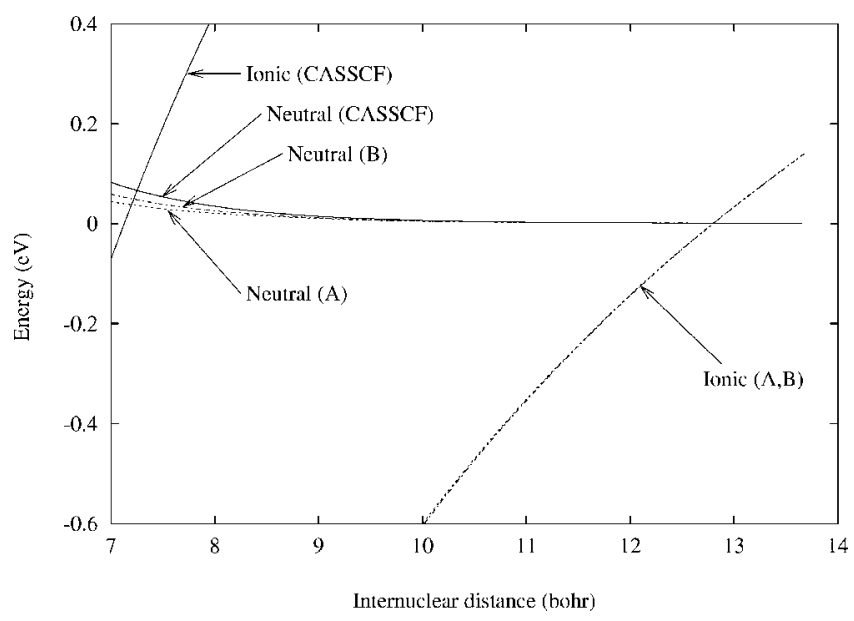

FIG. 5. Diabatic potential energy curves $(\mathrm{eV})$ for the neutral and ionic structures of $\mathrm{LiF}$ computed with the FDD-MR procedure at the CASSCF $2 / 2$ and SC-NEVPT2 level. A and B indicate different choices of $\gamma$ used for the construction of the matrix elements of the $\hat{\mathcal{H}}+\hat{\Delta}$ operator (see text). The zero of the energy is the energy of the neutral VB structure at $R$ $=100$ bohrs.

(unperturbed) wave function of the GS and of the orthogonal component $\Psi_{m}^{\perp}$ [see Eq. (12)]. We note here that this procedure cannot assure that this space is even qualitatively sufficient to describe both the GS and the first excited state: therefore the central aim is only to obtain a correct (decontracted) description of the GS. With this active space the two variants of NEVPT2 do not have the same behavior and therefore different choices of $\gamma$ values have been used: for PC-NEVPT2 the A and B choices used for the CASSCF $2 / 2$ case have been applied, while for SC-NEVPT2 we have considered the following choices:

(a) $\gamma=0.0, \pm 0.01 \rightarrow$ "C";

(b) $\gamma=0.0, \pm 0.02 \rightarrow$ "D";

(c) $\gamma=0.0, \pm 0.03 \rightarrow$ "E."

The origin of such a difference is described in the following.

The SC-NEVPT2 and PC-NEVPT2 results are reported in Figs. 6 and 7, respectively. One first notes that in this case

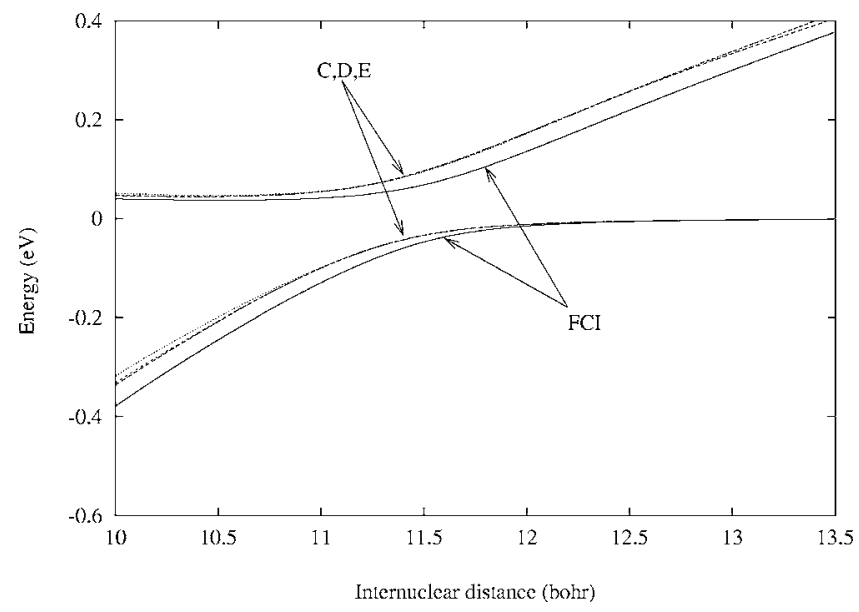

FIG. 6. Adiabatic potential energy curves (eV) for the first two ${ }^{1} \Sigma^{+}$states of $\mathrm{LiF}$ computed with the FDD-MR procedure at the CASSCF 6/6+SC-NEVPT2 level. C, D, and E indicate a decontraction based on different choices of $\gamma$ (see text). The three $\mathrm{C}, \mathrm{D}$, and $\mathrm{E}$ curves are almost indistinguishable in figure. The FCI curves are also reported for comparison.

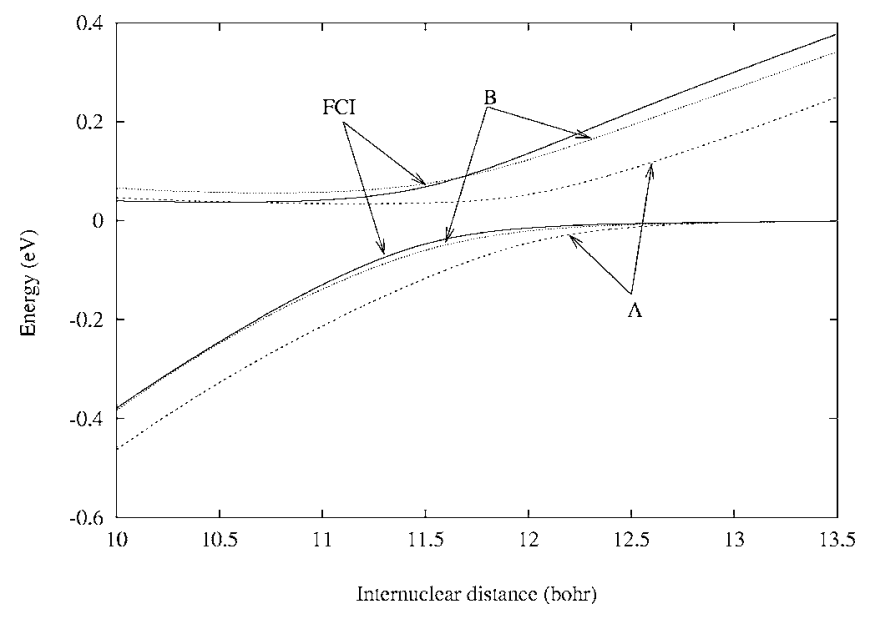

FIG. 7. Adiabatic potential energy curves (eV) for the first two ${ }^{1} \Sigma^{+}$states of $\mathrm{LiF}$ computed with the FDD-MR procedure at the CASSCF 6/6+PC-NEVPT2 level. A and B indicate a decontraction based on different choices of $\gamma$ (see text). The FCI curves are also reported for comparison.

the results are closer to the FCI description when compared with the CASSCF $2 / 2$ calculations. The three FDD-MR/SCNEVPT2 curves compare well with the FCI ones and are almost indistinguishable in the scale of Fig. 6. The use of smaller values for $\gamma$ leads to numerically unstable results with oscillating energy curves, while the use of larger values produces energy curves which depend on $\gamma$, thus indicating that with such values for $\gamma$ the hypothesis underlying the FDD-MR approach are not completely fulfilled. For the FDD-MR/PC-NEVPT2 approach the C, D and E choices of $\gamma$ values cannot be used because numerically unstable results are obtained. This is probably due to the greater numerical complexity of the PC-NEVPT2 variant with respect to the SC-NEVPT2 one. Indeed in a PC-NEVPT2 calculation the perturber functions are obtained by the diagonalization of a model Hamiltonian in a given set of spaces spanned by functions originated from the zero-order wave function and which can show linear dependencies. One has therefore to get rid of these linear dependencies before the diagonalization procedure and this is done by eliminating the eigenvectors of the overlap matrix associated to eigenvalues lower than a given (close to zero) threshold. This procedure, which is evidently prone to numerical instabilities for small perturbations of the zero-order wave functions and thus of the linear dependent functions defining the perturbers, is not needed in the SC-NEVPT2 variants, given that the perturber functions are in this case orthogonal by construction.

For these reasons the FDD-MR/PC-NEVPT2 approach has been applied with larger shifts $\gamma$, i.e., the $\mathrm{A}$ and $\mathrm{B}$ choices of $\gamma$ values used in the CASSCF $2 / 2$ case (see Fig. 7): the curves are in this case smoothly varying, but they show a dependence on the choice of the three values of $\gamma$ used in the FDD-MR approach. This is undeniably a nuisance but it can be essentially attributed to the numerical complexity of the PC-NEVPT2 approach more than to the FDD-MR technique. One has, however, to note that also in this case the agreement with the FCI curves is satisfactory for both choices of $\gamma$ values (even if the agreement is lower 
than in the FDD-MR/SC-NEVPT2 case) especially when compared with the bare SC or PC-NEVPT2 curves (Fig. 2).

We want to stress that the curves in Figs. 3-7 are obtained using only the wave function and energy of the GS (with three slightly different modifications of the composition of the wave function). Around the FCI avoided crossing these wave functions have essentially a neutral nature (as shown by Fig. 1) and it is worth noting that both the ground and the first excited states are in general correctly described by the diagonalization of the dressed Hamiltonian matrix.

\section{B. The $F_{2}$ molecule}

As a second case study, we have considered the GS of the $\mathrm{F}_{2}$ molecule. The accurate calculation of the GS potential energy curve of this molecule has been the subject of various studies. ${ }^{22,38-40,53,54}$ It has been shown that the dynamical polarization, i.e., the relaxation of the orbitals in each VB structure, plays a central role in the calculation of the dissociation energy. ${ }^{39}$ This effect is not included in a valence CASSCF wave function, given that the ionic and neutral forms are described using a unique set of orbitals. ${ }^{39,40}$ This leads to a destabilization of the ionic form, which at this level has too high an energy. Given the lowering of the ionic energy brought by the dynamical correlation one expects that the use of internally contracted multireference methods can be subjected to the bias introduced in the CASSCF wave function. It is, however, worth noting that in this case the problem manifests itself in a less dramatic way than in the case of LiF: in $F_{2}$ the ionic and neutral forms are rather far away in energy (the ionic form being higher in energy than the neutral one) for all internuclear distances $R$ and the two curves do not cross. Therefore the weight of the ionic form is expected to be always smaller than the one of the neutral form and to vary smoothly with $R$ (going to zero as $R$ increases).

In this case use has been made of the cc-pVQZ basis set, ${ }^{55}$ with the contraction scheme $(12 s 6 p 3 d 2 f 1 g) /$ $[5 s 4 p 3 d 2 f 1 g]$, keeping the spherical components. The calculations have been performed in the $D_{2 h}$ symmetry point group.

The CASSCF energies and wave functions are computed using the MOLCAS program package ${ }^{48}$ over quite a wide range of internuclear distances, from 2.10 to 10.0 bohrs (the energy for the two separated atoms is computed for $R$ $=100$ bohrs). Various active spaces have been considered: (a) the minimum valence active space, containing the two $2 p_{z}$ electrons and orbitals: CAS 2/2; (b) the CAS 2/2 space augmented with the two $2 p_{x}$ and the two $2 p_{y}$ electrons and orbitals: ten electrons in six orbitals, CAS 10/6; (c) the CAS $10 / 6$ space augmented with two correlating $2 p_{x}^{\prime}$ and two $2 p_{y}^{\prime}$ orbitals: CAS 10/10.

The zero-order CASSCF description is improved by correcting the energy to the second-order in perturbation using the SC and PC variants of the NEVPT approach and to the third-order with SC-NEVPT3 ${ }^{56}$ The FDD-MR decontraction algorithm is applied to SC-NEVPT2, PC-NEVPT2, and SCNEVPT3 using three zero-order CASCI wave functions computed applying three different one-electron energy shifts, $\gamma$, to the $b_{1 u} \sigma^{*}$ valence orbital. This allows us to build up a

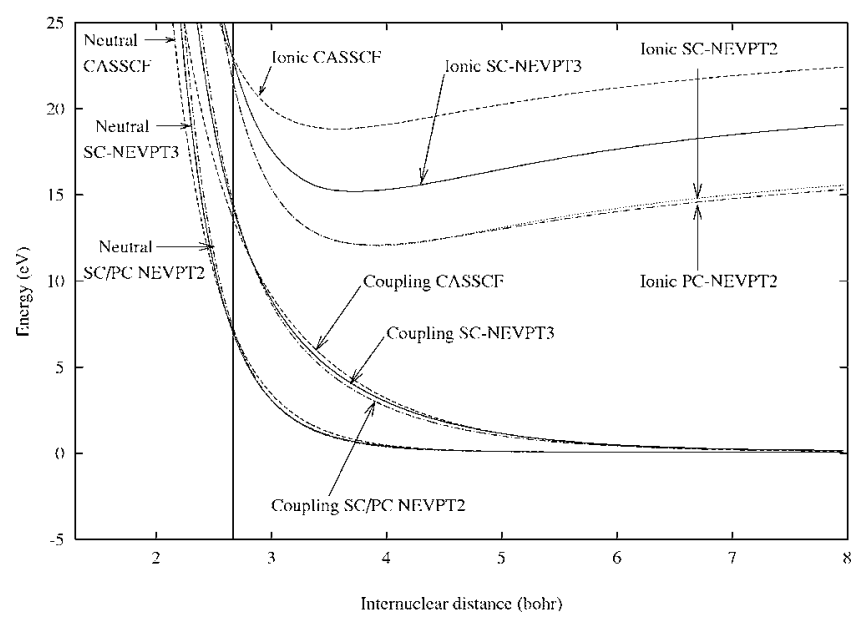

FIG. 8. Matrix elements of the Hamiltonian operator (at the CASSCF level) and of the $\hat{\mathcal{H}}+\hat{\Delta}$ operator (at the SC-NEVPT2, PC-NEVPT2, and SCNEVPT3 level) for the $\mathrm{F}_{2}$ molecule as a function of the internuclear distance. The diagonal elements are the energies of the neutral and of the ionic VB structures, indicated in the figure with Neutral and Ionic, respectively. The zero of the energy is the energy of the neutral VB structure at $R$ $=100.00$ bohrs. The off-diagonal element is the electronic coupling between the two structures and is reported without shift. The matrix elements of the dressing operator are obtained by shifting the one-electron energy of the $b_{1 u} \sigma^{*}$ valence orbital by $0,0.1$, and -0.1 hartree ("C" choice of $\gamma$ values, see text). The GS experimental equilibrium distance $(R=2.668$ bohrs $)$ is indicated with a vertical full line.

$2 \times 2$ dressing Hamiltonian. As in LiF, different choices for the three values $\gamma$ have been considered in order to test the stability of the decontraction algorithm:

(a) $\gamma=0.0, \pm 0.01 \rightarrow$ "A";

(b) $\gamma=0.0, \pm 0.05 \rightarrow$ "B";

(c) $\gamma=0.0, \pm 0.10 \rightarrow$ "C."

The basis on which the dressing Hamiltonian is built up is defined by the neutral (F..F) and ionic $\left(\mathrm{F}^{-} \mathrm{F}^{+}+\mathrm{F}^{+} \mathrm{F}^{-}\right)$forms (hereafter indicated with $|N\rangle$ and $|I\rangle$, respectively) in the case of the CAS 2/2 space and the $\Psi_{m}^{(0)}$ and $\Psi_{m}^{\perp}(\gamma)$ wave functions [Eq. (12) and Eqs. (18) and (19)] for the other active spaces. The neutral and ionic VB structures are written using orthogonal "atomic" orbitals (orbitals essentially centered on one atom, but with a tail on the other atom in order to guarantee the orthogonality constraint) obtained as the sum and the difference of the valence $\sigma_{g}$ and $\sigma_{u}$ molecular orbitals (MOs). They will be indicated in the following with $a$ and $b$. The VB structures here considered differ therefore from those of the standard VB approach (where strictly atomic, nonorthogonal, orbitals are used) and belong to the "orthogonalized VB" (OVB) family.

The CAS 2/2 results will first be examined. The use of such a small active space allows one to analyze the effect of the electronic correlation on the two relevant VB structures. In Fig. 8 the matrix elements of the Hamiltonian operator and of the FDD-MR dressed Hamiltonian operator (at the SC-NEVPT2, PC-NEVPT2, and SC-NEVPT3 level) on the $|N\rangle$ and $|I\rangle$ basis are reported for the $\mathrm{C}$ choice of $\gamma$ values (all energies are relative to the GS energy at dissociation). Let us first comment on some general characteristics of the curves. One notes that the energy curve for the neutral form is not strongly affected by the introduction of the dynamical correlation and that it is dissociative at all the levels of calculation 
TABLE III. $\mathrm{F}_{2}$ molecule: coefficient of the ionic $\left(C_{I}\right)$ and neutral $\left(C_{N}\right)$ structures in the GS wave function computed at different level of theory at the experimental equilibrium geometry ( $R=2.668 \mathrm{bohrs})$. For the FDD-MR/NEVPT2 and FDD-MR/NEVPT3 approaches, the values are reported for the SC variant (the PCNEVPT2 ones are in parentheses).

\begin{tabular}{lccc}
\hline \hline Method & $\left\langle\Psi^{\perp}(\gamma) \mid \Psi^{\perp}(-\gamma)\right\rangle$ & $C_{I}$ & $C_{N}$ \\
\hline CASSCF 2/2 & & 0.499 & 0.866 \\
CASSCF 2/2+S & & 0.546 & 0.838 \\
CASSCF 2/2+DDC2 & 0.560 & 0.829 \\
CASSCF 2/2+DDCI & 0.549 & 0.836 \\
CASSCF 2/2+SD & & 0.535 & 0.845 \\
FDD-MR(C)/NEVPT2 & & $0.529(0.529)$ & $0.849(0.849)$ \\
FDD-MR(C)/NEVPT3 & 0.510 & 0.861 \\
CASSCF 10/6 & & 0.501 & 0.865 \\
CASSCF 10/6+S & & 0.550 & 0.835 \\
CASSCF 10/6+DDC2 & & 0.543 & 0.840 \\
CASSCF 10/6+DDCI & & 0.539 & 0.843 \\
CASSCF 10/6+SD & & 0.538 & 0.843 \\
FDD-MR(C)/SC-NEVPT2 & & $0.529(0.529)$ & $0.847(0.847)$ \\
FDD-MR(C)/NEVPT3 & & 0.511 & 0.858 \\
CASSCF 10/10 & 0.980 & 0.537 & 0.824 \\
FDD-MR(C)/SC-NEVPT2 & 0.980 & $0.525(0.522)$ & $0.831(0.833)$ \\
FDD-MR(C)/NEVPT3 & & 0.524 & 0.831 \\
\hline \hline
\end{tabular}

here considered. The latter characteristic is also found in the $\mathrm{H}_{2}$ molecule using the OVB method ${ }^{57}$ and indicates that in the formation of the bond in $\mathrm{F}_{2}$ the ionic form plays a central role. The electronic coupling between the two VB structures is also very modestly affected by the introduction of the dynamical correlation at all values of $R$, apart from at very short internuclear distances. As expected, the ionic VB structure is higher in energy than the neutral one and correctly dissociates to two ionic atoms (the GS energy of $\mathrm{F}^{+} \mathrm{F}^{-}$depends on which of the two singlet states, ${ }^{1} D$ or ${ }^{1} S$, of the $\mathrm{F}^{+}$ atom is considered but a value between 16 and $20 \mathrm{eV}$ is reasonable). For $R$ larger than 3 bohrs, the effect of the dynamical correlation on this state is large and a remarkable lowering of the energy is found, with the SC/PC-NEVPT2 curves much lower than the SC-NEVPT3 one.

Concentrating our attention on the experimental equilibrium internuclear distance (for the sake of clarity Fig. 8 reports a vertical full line at this value of $R$ ), one promptly notes that the lowering of the ionic energy is negligible (with respect to the CAS value) at the third order and very small at the second order. This means that the matrix elements of the Hamiltonian in the $|N\rangle$ and $|I\rangle$ basis show modest variations (apart from a diagonal shift) passing from the CASSCF to the perturbative approaches, and the same happens therefore for the wave functions. One can therefore expect modest effects of the decontraction procedure on the dissociation energy and on the ionic to neutral ratio in the GS wave function at the equilibrium geometry. These considerations are confirmed by the data presented in Table III which reports the ionic $\left(C_{I}\right)$ and neutral $\left(C_{N}\right)$ coefficients for different wave functions computed at the experimental equilibrium geometry $(R=2.668$ bohrs $)$ for all the active spaces here considered. At the CASSCF $2 / 2$ level $C_{I}=0.499$, smaller than (but close to) the value obtained at higher levels of theory (also reported in Table III), such as, for instance, the
CASSCF plus single excitations CI $(\mathrm{CASSCF}+\mathrm{S})$, the $\mathrm{CASSCF}+$ difference dedicated CI (Ref. 21) (CASSCF +DDC2 and CASSCF+DDCI, and the CASSCF plus single and double excitations $\mathrm{CI}(\mathrm{CASSCF}+\mathrm{SD})$, for which $C_{I}$ ranges from $0.535(\mathrm{CASSCF}+\mathrm{SD})$ to 0.560 (CASSCF +DDC2) with 0.549 being the best estimate. All these CI calculations have been performed using the CASDI (Ref. 22) program. The application of the FDD-MR decontraction method to SC/PC-NEVPT2 and SC-NEVPT3 gives for $C_{I}$ a value of 0.529 (SC/PC-NEVPT2) and 0.510 (SC-NEVPT3) in agreement with the $\mathrm{CASSCF}+\mathrm{SD}$ value. For all active spaces only the results for the "C" choice of $\gamma$ values are reported given the low variability found for the $C_{I}$ and $C_{N}$ values with the $\gamma$ values used, thus showing a high stability of the results with respect to the numerical parameters.

The spectroscopic parameters $\left(D_{e}, R_{e}\right.$, and $\left.\Delta G_{1 / 2}\right)$, reported in Table IV (again only the $C$ choice of $\gamma$ values is reported for simplicity), also confirm what is said for the wave function. They are computed from the minimum of the spline fitting of the computed points $\left(D_{e}\right.$ and $\left.R_{e}\right)$ and by numerically solving the vibrational rotationless Schrödinger equation $\left(\Delta G_{1 / 2}\right)$. At the CASSCF $2 / 2$ level the value for $D_{e}$ $(0.720 \mathrm{eV})$ is largely underestimated (experimental ${ }^{63}$ $1.66 \mathrm{eV})$, while the second and third-order corrections are closer to the experimental value (with a modest overestimate and sizable underestimate, respectively). The CASSCF value for $\Delta G_{1 / 2}\left(663.5 \mathrm{~cm}^{-1}\right.$, experimental $\left.{ }^{64} 892 \mathrm{~cm}^{-1}\right)$ is improved by the introduction of the dynamical correlation, with the SC-NEVPT3 value differing from the experimental one by $\simeq 30 \mathrm{~cm}^{-1}$. A similar trend is observed for $R_{e}$.

The application of the FDD-MR approach leads to modest variations for $D_{e}$ (as expected from the previous considerations) and $R_{e}$ at the NEVPT2 level and very small variations for both quantities at the SC-NEVPT3 level. The 
TABLE IV. Spectroscopic constants of the $\mathrm{F}_{2}$ molecule. For the NEVPT2 and FDD-MR/NEVPT2 approach, the values are reported for the SCNEVPT2 variant (the PC-NEVPT2 ones are in parentheses). A selection of the previously published theoretical values is also reported.

\begin{tabular}{|c|c|c|c|}
\hline Method & $D_{e}(\mathrm{eV})$ & $R_{e}$ (bohr) & $\Delta G_{1 / 2}\left(\mathrm{~cm}^{-1}\right)$ \\
\hline \multicolumn{4}{|c|}{ CASSCF $2 / 2$} \\
\hline CASSCF & 0.720 & 2.776 & 663.5 \\
\hline NEVPT2 & $1.717(1.720)$ & $2.638(2.638)$ & $947.2(947.0)$ \\
\hline NEVPT3 & 1.390 & 2.678 & 861.6 \\
\hline FDD-MR(C)/NEVPT2 & $1.751(1.754)$ & $2.655(2.655)$ & $918.3(917.5)$ \\
\hline FDD-MR(C)/NEVPT3 & 1.395 & 2.683 & 849.0 \\
\hline \multicolumn{4}{|c|}{ CASSCF $10 / 6$} \\
\hline CASSCF & 0.768 & 2.767 & 679.8 \\
\hline NEVPT2 & $1.830(1.878)$ & $2.629(2.629)$ & $972.6(978.9)$ \\
\hline NEVPT3 & 1.476 & 2.674 & 877.1 \\
\hline FDD-MR(C)/NEVPT2 & $1.860(1.908)$ & $2.644(2.646)$ & $928.9(929.3)$ \\
\hline FDD-MR(C)/NEVPT3 & 1.481 & 2.678 & 860.5 \\
\hline \multicolumn{4}{|c|}{ CASSCF $10 / 10$} \\
\hline CASSCF & 1.515 & 2.678 & 884.3 \\
\hline NEVPT2 & $1.589(1.612)$ & $2.682(2.685)$ & $886.6(883.1)$ \\
\hline NEVPT3 & 1.604 & 2.674 & 893.0 \\
\hline FDD-MR(C)/NEVPT2 & $1.595(1.621)$ & $2.678(2.682)$ & $889.1(886.9)$ \\
\hline FDD-MR(C)/NEVPT3 & 1.612 & 2.670 & 895.6 \\
\hline$M P-E N^{a}$ & 1.77 & 2.666 & $924.4^{\mathrm{b}}$ \\
\hline$(\mathrm{SC})^{2} \mathrm{SDCI}^{\mathrm{c}}$ & 1.59 & 2.670 & $915.2^{\mathrm{b}}$ \\
\hline $\mathrm{MRCI}^{\mathrm{d}}$ & 1.62 & 2.668 & $921.2^{\mathrm{b}}$ \\
\hline $\operatorname{CCSD}(T)^{\mathrm{e}}$ & 1.56 & 2.663 & $955.5^{\mathrm{b}}$ \\
\hline Experimental & $1.66^{\mathrm{f}}$ & $2.668^{\mathrm{f}}$ & $892^{\mathrm{g}}$ \\
\hline
\end{tabular}

${ }^{\mathrm{a}}$ Second-order perturbation theory, Ref. 58 .

${ }^{\mathrm{b}}$ The value reported is for $\omega_{e}$ and not for $\Delta G_{1 / 2}$.

${ }^{\mathrm{c}}$ Size-consistent multireference CI, Ref. 59.

${ }^{\mathrm{d}}$ References 60 and 61.

${ }^{\mathrm{e}}$ Reference 62.

${ }^{\mathrm{f}}$ Reference 63.

${ }^{\mathrm{g}}$ Raman Spectroscopy, Ref. 64.

variations found for $\Delta G_{1 / 2}$ are more sizable for NEVPT2 $\left(\simeq 30 \mathrm{~cm}^{-1}\right)$ and also for SC-NEVPT3 are not negligible $\left(\simeq 12 \mathrm{~cm}^{-1}\right)$.

With respect to the CAS $2 / 2$ results, the use of a slightly modified active space (CAS 10/6) only slightly modifies the description, the main difference being in the improved agreement of the SC-NEVPT3 values for $D_{e}$ and $\Delta G_{1 / 2}$ with the experimental ones. The agreement is further improved with the largest active space (CASSCF 10/10) where the differences with the experimental values are always small and particularly small at the highest level, FDD-MR/SC-NEVPT3, where they are $0.05 \mathrm{eV}$ for $D_{e}, 0.002 \mathrm{bohr}$ for $R_{e}$, and $4 \mathrm{~cm}^{-1}$ for $\Delta G_{1 / 2}$.

For the sake of completeness the potential energy curve of the ground state for some relevant approaches (CASSCF, NEVPT2, FDD-MR/NEVPT2, NEVPT3, and FDD-MR/ NEVPT3 with the $2 / 2$ active space and NEVPT3 with the 10/10 active space) is reported in Fig. 9, from which one can note that the effect of the decontraction procedure is more pronounced in an interval of $R$ larger than the equilibrium internuclear distance, both for the NEVPT2 $2 / 2$ and NEVPT3 2/2 approaches. The best curve obtained in this

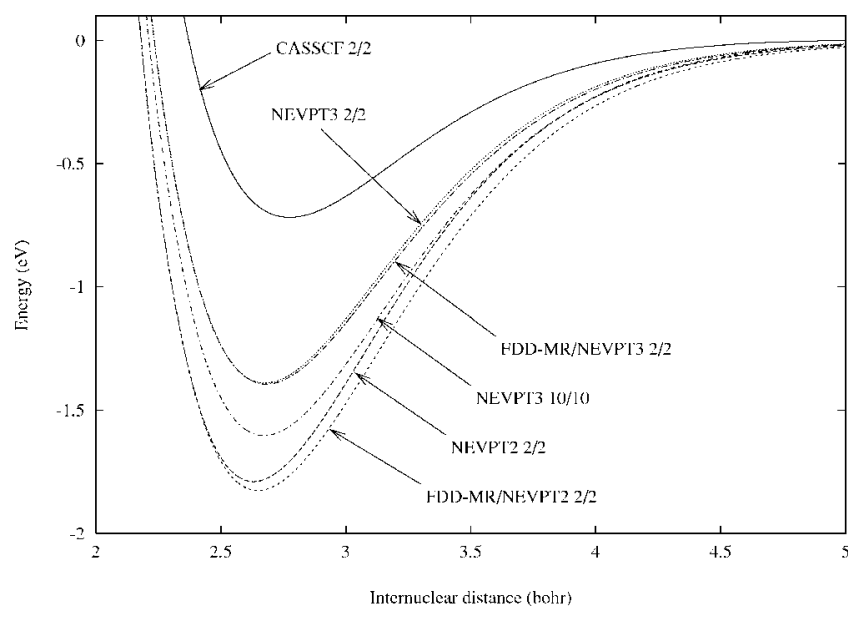

FIG. 9. Potential energy curves for the ${ }^{1} \Sigma_{g}^{+}$ground state of the $\mathrm{F}_{2}$ molecule computed at the CASSCF, NEVPT2, FDD-MR/NEVPT2, NEVPT3, and FDD-MR/NEVPT3 level with the $2 / 2$ active space and at the NEVPT3 level with the 10/10 active space. All NEVPT values are for the strongly contracted variant. The FDD-MR results are obtained by shifting the oneelectron energy of the $b_{1 u} \sigma^{*}$ orbital by $0,0.1$, and -0.1 hartree ("C" choice of $\gamma$ values, see text).

work (NEVPT3 10/10, almost indistinguishable from the FDD-MR/NEVPT3 10/10 one) is reported for comparison.

From Table III one notes that when Eqs. (12), (18), and (19) are used (CAS $10 / 6$ and 10/10) the overlap $\left\langle\Psi^{\perp}(\gamma) \mid \Psi^{\perp}(-\gamma)\right\rangle$ is always close to one (for the A and B choices of $\gamma$ values the overlap is higher than with the $\mathrm{C}$ one), thus confirming the hypothesis of the invariance of the $\Psi^{\perp}$ wave function with respect to small variations of $\gamma$.

From these results one can conclude that the ionic/ neutral mixing at the equilibrium geometry is not the source for the large deviations sometimes found in the calculation of the dissociation energy of the $\mathrm{F}_{2}$ molecule. Modest variations on this value have been found by internally decontracting the zero-order wave function. On the other hand for values of $R$ slightly larger than the equilibrium one, the modification due to the internal decontraction should be more pronounced (due to the lowering of the ionic energy). The fact that $\Delta G_{1 / 2}$ is more affected by the internal decontraction than $D_{e}$ and $R_{e}$ agrees with this consideration. Finally, for large values of $R$ the effect of the internal decontraction becomes vanishing given that the electronic coupling rapidly goes to zero.

Other properties of the $F_{2}$ molecule can be more strongly affected by the internal decontraction of the GS wave function, an example being the calculation of the emission intensity from the $C^{1} \Sigma_{u}$ excited states. Close to its equilibrium geometry (experimental ${ }^{65} R_{e}=3.619 \mathrm{bohr}$ ) this state is known to arise essentially from the $2 p \sigma_{g}^{1} \rightarrow 2 p \sigma_{u}^{1}$ excitation and to have ionic character. ${ }^{66}$ Using the simplest description of this state (CAS 2/2), one can easily demonstrate that the emission intensity is roughly proportional to the square of the coefficient of the ionic VB structure in the GS wave function. Indeed the $C^{1} \Sigma_{u}$ state is qualitatively described, for the two valence electrons, by the spatial wave function $(a a-b b) / \sqrt{2}$ ( $a$ and $b$ being the orthogonal $2 p_{z}$ atomic orbitals centered on the two $\mathrm{F}$ atoms) and for this wave function the matrix element of the electric dipole operator is nonvanishing only with the ionic ${ }^{1} \Sigma_{g}$ VB structure 
TABLE V. Matrix elements of the Hamiltonian operator (at the CASSCF level) and of the dressing operator (at the SC-NEVPT2, PC-NEVPT2, and SC-NEVPT3 level) on the ionic and neutral structures for the $\mathrm{F}_{2}$ molecule for two relevant internuclear distances. The matrix elements of the dressing operator are obtained by shifting the one-electron energy of the $b_{1 u} \sigma^{*}$ orbital by $0,0.1$, and -0.1 hartree $(C$ choice of $\gamma$ values, see text). Energies in hartree, and distance in bohr.

\begin{tabular}{|c|c|c|c|c|c|}
\hline Method & $\langle N|\hat{\mathcal{H}}| N\rangle$ & $\langle I|\hat{\mathcal{H}}| I\rangle$ & $\langle N|\hat{\mathcal{H}}| I\rangle$ & $C_{I}$ & $C_{N}$ \\
\hline \multicolumn{6}{|c|}{ GS experimental equilibrium geometry $(R=2.668$ bohrs $)$} \\
\hline CASSCF & -198.553770 & -197.972084 & -0.502061 & 0.499 & 0.866 \\
\hline FDD-MR(C)/SC-NEVPT2 & -199.063555 & -198.535873 & -0.540252 & 0.530 & 0.848 \\
\hline FDD-MR(C)/SC-NEVPT3 & -199.088745 & -198.501902 & -0.534149 & 0.509 & 0.861 \\
\hline \multicolumn{6}{|c|}{$C^{1} \Sigma_{u}$ experimental equilibrium geometry $(R=3.619$ bohrs $)$} \\
\hline CASSCF & -198.783362 & -198.126693 & -0.173306 & 0.240 & 0.971 \\
\hline FDD-MR(C)/SC-NEVPT2 & -199.306423 & -198.885615 & -0.151038 & 0.306 & 0.952 \\
\hline FDD-MR(C)/SC-NEVPT3 & -199.324060 & -198.797698 & -0.161444 & 0.272 & 0.962 \\
\hline
\end{tabular}

(being proportional to $\langle a|z| a\rangle-\langle b|z| b\rangle$ ). The application of the FDD-MR approach to the GS wave function at $R$ $=3.619$ bohrs results in an increase of the ionic coefficient from 0.240 in the CASSCF wave function to 0.304 at the SC- and PC-NEVPT2 level and to 0.272 at the SC-NEVPT3 level. The transition electric dipole is therefore increased by $26.7 \%$ and $13.3 \%$ at the second and third orders, respectively, implying an increase for the emission intensity of $60.4 \%$ and $28.4 \%$, respectively. In this case the internal decontraction of the GS wave function has a sizable effect on the studied property. For the sake of clarity the matrix elements of $\hat{\mathcal{H}}$ and $\hat{\Delta}$ (for the $C$ choice of $\gamma$ values) are reported in Table $\mathrm{V}$ for the two relevant geometries here discussed (experimental equilibrium geometry of the GS and of the $C{ }^{1} \Sigma_{u}$ state).

It may be interesting to see whether the neutral and ionic VB configurations defined from our diabatization have a physical significance. To this aim, one can compare the ionic curve reported in Fig. 8 with the $C^{1} \Sigma_{u}$ excited state of $\mathrm{F}_{2}$. Clearly the ionic configuration reported in Fig. 8 is of ${ }^{1} \Sigma_{g}$ type and therefore it cannot be directly compared with the $C^{1} \Sigma_{u}$ state, but one can note that in a simplified model where only the two valence electrons are considered, the ionic ${ }^{1} \Sigma_{g}$ configuration can be described (disregarding the spin) by the wave function $(a a+b b) / \sqrt{2}$. In this model, the ionic ${ }^{1} \Sigma_{g}$ configuration and the $C{ }^{1} \Sigma_{u}$ state are separated in energy by $2 K_{a b}$, where $K_{a b}$ is the exchange integral for the two orbitals $a$ and $b$. This separation can be supposed to be small with respect to the energy difference between the GS and the $C^{1} \Sigma_{u}$ excited state. This hypothesis is confirmed by the calculation of $2 K_{a b}$ using the CASSCF $2 / 2$ orbitals of the ground state. Noting that

$$
2 K_{a b}=\frac{\left\langle\sigma_{g} \sigma_{g} \mid \sigma_{g} \sigma_{g}\right\rangle+\left\langle\sigma_{u} \sigma_{u} \mid \sigma_{u} \sigma_{u}\right\rangle}{2}-\left\langle\sigma_{g} \sigma_{u} \mid \sigma_{g} \sigma_{u}\right\rangle,
$$

one has that $2 K_{a b}$ is less than $0.5 \mathrm{eV}$ at the GS equilibrium geometry and rapidly goes to zero if $R$ increases.

By shifting the ${ }^{1} \Sigma_{g}$ NEVPT curves by $2 K_{a b}$ [computed with Eq. (22)] one has $R_{e}=3.90,3.87,3.69$ bohrs, $E\left(R_{e}\right)$ $=11.92,11.96,15.05 \mathrm{eV}$, vertical excitation energy $=21.22,21.23,22.47 \mathrm{eV}$, and energy difference with the GS at its equilibrium geometry $=11.62,11.62,14.49 \mathrm{eV}$ for SCNEVPT2, PC-NEVPT2, and SC-NEVPT3, respectively.
These curves compare quite well with the one reported in Ref. 66 for the $C^{1} \Sigma_{u}$ state $\left(T_{0}=11.57 \mathrm{eV}, R_{e}=3.62\right.$ bohrs $)$, showing a similar shape and being in the same energy region.

We stress again that in the present calculation the ionic VB energy is obtained by performing calculations only on the GS and a qualitative agreement for an excited state has to be considered satisfactory.

A similar comparison can be done between the neutral curve of Fig. 8 and the first ${ }^{3} \Sigma_{u}^{+}$state, which is purely neutral and qualitatively described by the spatial wave function $(a b-b a) / \sqrt{2}$. Again the two wave functions have different spatial symmetry and in this case also different spin multiplicity, but in the simplest model they are again separated by $2 K_{a b}$, so they are expected to be close and to have quite a similar shape. The ${ }^{3} \Sigma_{u}^{+}$state is found ${ }^{67}$ to be dissociative and placed $7.5 \mathrm{eV}$ higher than the GS at the GS equilibrium geometry. The neutral curves reported in Fig. 8 agree with these results, the vertical excitation energy being $7.34 \mathrm{eV}$ (SC/PC-NEVPT2) and $7.04 \mathrm{eV}$ (SC-NEVPT3), while for the ${ }^{3} \Sigma_{u}^{+}$state (computed by shifting the neutral ${ }^{1} \Sigma_{g}^{+}$curves by $\left.2 K_{a b}\right)$ the vertical excitation energy is $6.91 \mathrm{eV}$ (SC/PCNEVPT2) and $6.62 \mathrm{eV}$ (SC-NEVPT3).

It is quite gratifying to see that our analysis of the ground state wave function and state-specific diabatization furnishes physically relevant information about the position of excited states (of other symmetries).

\section{DISCUSSION}

The comparison between the $\mathrm{LiF}$ and the $\mathrm{F}_{2}$ problems permits a discussion about the importance of the decontraction. The decontraction produces a component in the reference space, which can be assimilated to the second-order contribution to the wave function expansion

$$
\begin{aligned}
P_{0}\left|\Psi_{m}^{(2)}\right\rangle & =\sum_{n \neq m} \sum_{\alpha \notin S_{0}}\left|\Psi_{n}^{(0)}\right\rangle \frac{\left\langle\Psi_{n}^{(0)}|\hat{\mathcal{H}}| \alpha\right\rangle\left\langle\alpha|\hat{\mathcal{H}}| \Psi_{m}^{(0)}\right\rangle}{\left(E_{m}^{(0)}-E_{n}^{(0)}\right)\left(E_{m}^{(0)}-E_{\alpha}^{(0)}\right)} \\
& =\sum_{n \neq m}\left|\Psi_{n}^{(0)}\right\rangle \frac{\left\langle\Psi_{n}^{(0)}|\hat{\mathcal{H}}| \Psi_{m}^{(1)}\right\rangle}{E_{m}^{(0)}-E_{n}^{(0)}}
\end{aligned}
$$

Analogously, the decontraction contribution to the energy can be supposed to be similar to a component of the fourth- 
order energy correction

$$
E_{m}^{(4)}=\sum_{\beta \notin S_{0}} \sum_{n \neq m} \sum_{\alpha \notin S_{0}} \frac{\left\langle\Psi_{m}^{(0)}|\hat{\mathcal{H}}| \beta\right\rangle\left\langle\beta|\hat{\mathcal{H}}| \Psi_{n}^{(0)}\right\rangle\left\langle\Psi_{n}^{(0)}|\hat{\mathcal{H}}| \alpha\right\rangle\left\langle\alpha|\hat{\mathcal{H}}| \Psi_{m}^{(0)}\right\rangle}{\left(E_{m}^{(0)}-E_{\beta}^{(0)}\right)\left(E_{m}^{(0)}-E_{n}^{(0)}\right)\left(E_{m}^{(0)}-E_{\alpha}^{(0)}\right)} .
$$

These corrections are of course expected to be crucial when there exists at least a state $\Psi_{n}^{(0)}$ close in energy to $\Psi_{m}^{(0)}$ and strongly interacting with $\Psi_{m}^{(1)}$. The existence of a near degeneracy in the reference space is a case where decontraction is highly recommended.

It may be interesting as well to consider the problem from a VB point of view, starting with the $\mathrm{LiF}$ and $\mathrm{F}_{2}$ systems. Both problems can be understood in terms of an interaction between two VB configurations, a neutral singlet and an ionic configuration, $\mathrm{Li}^{+} \mathrm{F}^{-}$or $\left(\mathrm{F}^{-} \mathrm{F}^{+}+\mathrm{F}^{+} \mathrm{F}^{-}\right) / \sqrt{2}$. In both molecules the dynamical correlation is stronger in the ionic configuration than in the neutral one, by $\simeq 2 \mathrm{eV}$ in $\mathrm{LiF}$ and up to $5 \mathrm{eV}$ in $\mathrm{F}_{2}$ beyond 3.5 bohrs. This differential dynamical correlation effect is crucial in both cases, it is responsible for the shift of the avoided crossing to large interatomic distance in $\mathrm{LiF}$ and for half of the binding energy in $\mathrm{F}_{2}$, but the decontraction is necessary in $\mathrm{LiF}$ while it plays a minor role in $F_{2}$ near its equilibrium geometry. The difference may of course be related to the near degeneracy in LiF, while the energy separation between the ionic and neutral VB components is large in $\mathrm{F}_{2}$. It is nevertheless interesting to compare the contracted and decontracted treatments in such problems. In terms of the elements of the dressing operator $\hat{\Delta}$, for the GS,

$$
\left|\Psi_{m}^{(0)}\right\rangle=\lambda|N\rangle+\mu|I\rangle
$$

the contracted second-order correction is

$$
E_{m}^{(2)}=\lambda^{2} \Delta_{N N}+\mu^{2} \Delta_{I I}+2 \lambda \mu \Delta_{N I} .
$$

Expressing $\hat{\Delta}$ in the basis of $\Psi_{m}^{(0)}$ and $\Psi^{\perp}$

$$
\Psi^{\perp}=-\mu|N\rangle+\lambda|I\rangle,
$$

one gets

$$
\begin{aligned}
& \left\langle\Psi_{m}^{(0)}|\hat{\Delta}| \Psi^{\perp}\right\rangle=\lambda \mu\left(\Delta_{I I}-\Delta_{N N}\right)+\left(\lambda^{2}-\mu^{2}\right) \Delta_{N I}, \\
& \left\langle\Psi^{\perp}|\hat{\Delta}| \Psi^{\perp}\right\rangle=\mu^{2} \Delta_{N N}+\lambda^{2} \Delta_{I I}-2 \lambda \mu \Delta_{N I} .
\end{aligned}
$$

The effect of the decontraction can be estimated from a second-order expansion

$$
\begin{aligned}
\delta E_{m}^{(2)} & =\frac{\left\langle\Psi_{m}^{(0)}|\hat{\Delta}| \Psi^{\perp}\right\rangle^{2}}{E_{m}^{(0)}+\left\langle\Psi_{m}^{(0)}|\hat{\Delta}| \Psi_{m}^{(0)}\right\rangle-E^{\perp}-\left\langle\Psi^{\perp}|\hat{\Delta}| \Psi^{\perp}\right\rangle} \\
& =\frac{\left[\lambda \mu\left(\Delta_{I I}-\Delta_{N N}\right)+\left(\lambda^{2}-\mu^{2}\right) \Delta_{N I}\right]^{2}}{E_{m}^{(0)}-E^{\perp}+\left(\lambda^{2}-\mu^{2}\right)\left(\Delta_{N N}-\Delta_{I I}\right)+4 \lambda \mu \Delta_{N I}} .
\end{aligned}
$$

In the simplifying hypothesis where the dressing matrix reduces to the $\Delta_{\text {II }}$ element one has

$$
E_{m}^{(2)}=\mu^{2} \Delta_{I I},
$$

$$
\delta E_{m}^{(2)}=\frac{\lambda^{2} \mu^{2} \Delta_{I I}^{2}}{E_{m}^{(0)}-E^{\perp}-\left(\lambda^{2}-\mu^{2}\right) \Delta_{I I}} .
$$

The ratio between the decontraction effect and the contracted second-order energy,

$$
\frac{\delta E_{m}^{(2)}}{E_{m}^{(2)}}=\frac{\lambda^{2} \Delta_{I I}}{E_{m}^{(0)}-E^{\perp}-\left(\lambda^{2}-\mu^{2}\right) \Delta_{I I}},
$$

can be very important when the dressing strongly reduces the amplitude of the energy denominator. This will be the case in magnetic diradical systems, which are not degenerate $(|\lambda|$ $\gg|\mu|)$. The dynamical correlation strongly reduces the effective energy of the ionic VB component (for instance, from 25 to $7 \mathrm{eV}$, see Ref. 68) and the effect of the decontraction may be of the same order of magnitude as the second-order contracted correction. A forthcoming work will be devoted to this problem.

\section{CONCLUSION}

The present paper has recalled the difficulties met by the internally contracted multireference procedures, despite the computational benefit of the internal contraction. Some of the spurious effects induced by the internal contraction (in the case of interaction between close lying states, or of weakly avoided crossings) can be solved by the construction of low dimensional effective Hamiltonians ${ }^{11,32,33}$ through multistate extensions of these algorithms. However, these solutions do not allow one to obtain continuous potential energy surfaces when one cannot identify a closed set of states which do not exhibit curve crossings with external states. Moreover they cannot be applied in many problems where it is not possible to improve the quality of the component of the desired wave function in the reference space by a rotation with a spectroscopically identifiable and computationally accessible excited state. The case of the binuclear magnetic complexes, where the ionic VB component of the singlet state is grossly underestimated at the valence CAS level, is a well documented example. ${ }^{36,68}$

The paper has proposed a state-specific decontraction procedure for state-specific MR algorithms. It is based on the use of slightly perturbed zero-order wave functions. In a certain sense it may be compared to finite-field computations of dipole moments or other observables, but it goes through the construction of an effective Hamiltonian in the reference space. The dressing matrix, reflecting the effect of the interaction with the outer space, is state-specific and the final effective Hamiltonian is an intermediate Hamiltonian. With a suitable choice of the components on which the wave function is expanded, the method provides an orthogonal valence 
bond reading of the state of interest. The effective valence Hamiltonian built on such basis gives excited roots which have been shown to be relevant.

The method would be rigorous if the outer space vectors were not affected by the changes of $\Psi_{m}^{(0)}$. Although this is not strictly true for externally contracted procedures, one may apply the method to such algorithms and one may test the numerical stability of the method by performing more calculations than the strict minimal number.

The method has been tested in the frame of the statespecific version of the NEVPT algorithm, which is both internally and externally contracted and the results have shown the potentialities of the FDD-MR method. On the LiF molecule it has been possible to remove the totally unphysical behavior of the internally contracted approach and to obtain a good quality potential energy curve for the ground state. For $\mathrm{F}_{2}$ the FDD-MR approach has led to a better understanding of the physics of bond formation, clarifying that the neutral/ionic ratio is well represented at the valence CASSCF level. It has been shown that the FDD-MR approach is not crucial for this molecule for the calculation of the spectroscopic constants. The use of a large active space in the CASSCF calculation, of a high level treatment of the electronic correlation (SC-NEVPT3) and of the FDD-MR procedure has allowed one to obtain a very close agreement of the computed value of some spectroscopic constants with the experimental findings. On the contrary the emission intensity for the radiative transition from the excited $C^{1} \Sigma_{u}$ state to the GS has been shown to depend on the internal contraction/decontraction of the GS wave function. Finally, for both molecules the use of the FDD-MR approach has made it possible to describe, at a qualitative $\left(\mathrm{F}_{2}\right)$ or more than qualitative $(\mathrm{LiF})$ level, one or two excited states also. This result is noteworthy because the procedure here presented is based on a small number of calculations on the ground state.

Further work ${ }^{37}$ will present applications of the NEVPT2 method, as modified by the decontraction, to magnetic systems with comparison of the Effective Hamiltonians obtained with the present method with those which are rigorously established from variational CI calculations through Bloch's theory.

\section{ACKNOWLEDGMENTS}

This work has been financed by the University of Ferrara (nano \& nano project) and by the Italian MIUR through its PRIN funds. The "Laboratoire de Physique Quantique" is "Unite Mixte de Recherche" (UMR5626) of the CNRS.

${ }^{1}$ D. J. Rowe, Rev. Mod. Phys. 40, 153 (1968).

${ }^{2}$ D. L. Yeager and P. Jørgensen, Chem. Phys. Lett. 65, 77 (1979).

${ }^{3}$ E. Dalgaard, J. Chem. Phys. 72, 816 (1980).

${ }^{4}$ H. Koch, O. Christiansen, P. Jørgensen, A. M. Sanchez de Merás, and T. Helgaker, J. Chem. Phys. 106, 1808 (1997).

${ }^{5}$ B. Huron, J.-P. Malrieu, and P. Rancurel, J. Chem. Phys. 58, 5745 (1973).

${ }^{6}$ R. Cimiraglia, Int. J. Quantum Chem. 60, 167 (1996).

${ }^{7}$ C. Angeli and M. Persico, Theor. Chem. Acc. 98, 117 (1997).

${ }^{8}$ K. Hirao, Chem. Phys. Lett. 190, 374 (1992).

${ }^{9}$ P. M. Kozłowski and E. R. Davidson, J. Chem. Phys. 100, 3672 (1994).
${ }^{10}$ K. Andersson, P.-Å. Malmqvist, and B. O. Roos, J. Chem. Phys. 96, 1218 (1992).

${ }^{11}$ H. Nakano, J. Chem. Phys. 99, 7983 (1993).

${ }^{12}$ C. Angeli, R. Cimiraglia, S. Evangelisti, T. Leininger, and J.-P. Malrieu, J. Chem. Phys. 114, 10252 (2001)

${ }^{13}$ C. Angeli, R. Cimiraglia, and J.-P. Malrieu, Chem. Phys. Lett. 350, 297 (2001)

${ }^{14}$ C. Angeli, R. Cimiraglia, and J.-P. Malrieu, J. Chem. Phys. 117, 9138 (2002)

${ }^{15}$ U. S. Mahapatra, B. Datta, and D. Mukherjee, Chem. Phys. Lett. 299, 42 (1999).

${ }^{16}$ P. Ghosh, S. Chattopadhyay, D. Jana, and D. Mukherjee, Int. J. Mol. Sci. 3, 733 (2002)

${ }^{17}$ F. Neese, J. Chem. Phys. 119, 9428 (2003).

${ }^{18}$ P. Celani, H. Stoll, H.-J. Werner, and P. J. Knowles, Mol. Phys. 102, 2369 (2004)

${ }^{19}$ Z. Rolik, Á. Szabados, and P. R. Surján, J. Chem. Phys. 119, 1922 (2003).

${ }^{20}$ H. J. Werner and P. J. Knowles, J. Chem. Phys. 89, 5803 (1988).

${ }^{21}$ J. Mirales, O. Castell, R. Caballol, and J.-P. Malrieu, Chem. Phys. 172, 33 (1993)

${ }^{22}$ N. Ben Amor and D. Maynau, Chem. Phys. Lett. 286, 211 (1998).

${ }^{23}$ P. Fulde and H. Stoll, J. Chem. Phys. 97, 4185 (1992).

${ }^{24}$ J.-P. Malrieu, J.-P. Daudey, and R. Caballol, J. Chem. Phys. 101, 8908 (1994).

${ }^{25}$ P. Mach, J. Mášik, J. Urban, and I. Hubač, Mol. Phys. 94, 173 (1998).

${ }^{26}$ J. Meller, J.-P. Malrieu, R. Caballol, J. Chem. Phys. 104, 4068 (1996).

${ }^{27}$ U. S. Mahapatra, B. Datta, and D. Mukherjee, Mol. Phys. 94, 157 (1998).

${ }^{28}$ I. Lindgren, J. Phys. B 7, 2441 (1974)

${ }^{29}$ C. Bloch, Nucl. Phys. 6, 329 (1958).

${ }^{30}$ J.-P. Malrieu Ph. Durand, and J.-P. Daudey, J. Phys. A 18, 809 (1985).

${ }^{31}$ E. R. Davidson, L. B. McMurchie, and S. J. Day, J. Chem. Phys. 74, 5491 (1981).

${ }^{32}$ F. Spiegelmann and J.-P. Malrieu, J. Phys. B 17, 1235 (1984).

${ }^{33}$ F. Spiegelmann and J.-P. Malrieu, J. Phys. B 17, 1259 (1984)

${ }^{34}$ J. Finley, P.-Å. Malmqvist, B. O. Roos, and L. Serrano Andrés, Chem. Phys. Lett. 288, 299 (1998).

${ }^{35}$ C. Angeli, S. Borini, M. Cestari, and R. Cimiraglia, J. Chem. Phys. 121, 4043 (2004)

${ }^{36}$ C. J. Calzado, J. Cabrero, J.-P. Malrieu, and R. Caballol, J. Chem. Phys. 116, 2728 (2002).

${ }^{37}$ C. J. Calzado, C. Angeli, R. Cimiraglia, and J.-P. Malrieu (unpublished).

${ }^{38}$ P. C. Hiberty, J. P. Flament, and E. Noizet, Chem. Phys. Lett. 189, 259 (1992).

${ }^{39}$ R. Ghailane, M.-B. Lepetit, and J.-P. Malrieu, J. Phys. Chem. 97, 94 (1993).

${ }^{40}$ P. C. Hiberty, S. Humbel, C. P. Byrman, and J. H. van Lenthe, J. Chem. Phys. 101, 5969 (1994).

${ }^{41}$ R. Cimiraglia, J. Chem. Phys. 83, 1746 (1985).

${ }^{42}$ J.-P. Malrieu, J.-L. Heully, and A. Zaitsevskii, Theor. Chim. Acta 90, 167 (1995).

${ }^{43}$ M. Persico, The Encyclopedia of Computational Chemistry (John Wiley \& Sons, Chichester, 1998), p. 852.

${ }^{44}$ C. W. Bauschlicher and S. R. Langhoff, J. Chem. Phys. 89, 4246 (1988).

${ }^{45}$ Ö. Legeza, J. Röder, and B. A. Hess, Mol. Phys. 101, 2019 (2003).

${ }^{46}$ J. Finley, P.-A. Malmqvist, B. O. Roos, and L. Serrano-Andrés, Chem. Phys. Lett. 288, 299 (1998).

${ }^{47}$ J. P. Finley and H. A. Witek, J. Chem. Phys. 112, 3958 (2000).

${ }^{48}$ K. Andersson, M. Barysz, A. Bernhardsson et al., MOLCAs, Version 5.4, Lund University, Sweden, 2002.

${ }^{49}$ R. Cimiraglia, J.-P. Malrieu, M. Persico, and F. Spiegelmann, J. Phys. B 18, 3073 (1985).

${ }^{50}$ M. Persico, R. Cimiraglia, and F. Spiegelmann, in Strategies and Applications in Quantum Chemistry, edited by Y. Ellinger and M. Defranceschi (Kluwer Academic, Dordrecht, 1996), p. 349.

${ }^{51}$ C. Sousa, D. Domíniguez, C. de Graaf, and F. Illas, J. Chem. Phys. 113, $9940(2000)$

${ }^{52}$ L. Serrano-Andrés, M. Merchán, and R. Lindh, J. Chem. Phys. 122, 104107 (2005).

${ }^{53}$ J. Pittner, J. Šmydke, P. Čársky, and I. Hubač, J. Mol. Struct.: THEOCHEM 547, 239 (2001).

${ }^{54}$ L. E. Forslund and N. Kaltsoyannis, New J. Chem. 27, 1108 (2003).

${ }^{55}$ T. H. Dunning, J. Chem. Phys. 90, 1007 (1989). 
${ }^{56}$ C. Angeli, B. Bories, A. Cavallini, and R. Cimiraglia, J. Chem. Phys. 124, 054108 (2006).

${ }^{57}$ C. Angeli, R. Cimiraglia, J.-P. Malrieu, J. Chem. Ed. (submitted).

${ }^{58}$ C. Angeli, R. Cimiraglia, and J.-P. Malrieu, Chem. Phys. Lett. 317, 472 (2000).

${ }^{59}$ J. Sanchez-Marin, I. Nebot-Gil, D. Maynau, and J.-P. Malrieu, Theor. Chim. Acta 92, 241 (1995).

${ }^{60}$ H. J. Werner and P. J. Knowles, Theor. Chim. Acta 78, 175 (1990).

${ }^{61}$ C. Hampel, K. A. Peterson, and H. J. Werner, Chem. Phys. Lett. 190, 1 (1992)

${ }^{62}$ K. A. Peterson, A. K. Wilson, D. E. Woon, and T. H. Dunning, Jr., Theor. Chem. Acc. 97, 251 (1997).
${ }^{63}$ K. P. Huber and G. Herzberg, Constants of Diatomic Molecules, Molecular Spectra and Molecular Structure IV (Van Nostrand Reinhold, New York, 1979).

${ }^{64}$ D. Andrychuk, J. Chem. Phys. 18, 233 (1951).

${ }^{65}$ E. A. Colbourn, M. Dagenais, A. E. Douglas, and A. W. Raymonda, Can. J. Phys. 54, 1343 (1975).

${ }^{66}$ H. Moriyama and Y. Wasada-Tsutsui, J. Chem. Phys. 118, 5413 (2003).

${ }^{67}$ I. A. Delyagina, D. B. Kokh, and A. M. Pravilov, J. Chem. Phys. 94, 170 (2003).

${ }^{68}$ C. J. Calzado, J. Cabrero, J.-P. Malrieu, and R. Caballol, J. Chem. Phys. 116, 3985 (2002) 